\title{
A tropical cyclone removal technique based on potential vorticity inversion to better quantify tropical cyclone contribution to the background circulation
}

\author{
Sho Arakane ${ }^{1,2}$ [D Huang-Hsiung $\mathrm{Hsu}^{1}$
}

Received: 27 April 2019 / Accepted: 3 February 2020 / Published online: 12 February 2020

(c) The Author(s) 2020

\begin{abstract}
An effective way of investigating the effects of tropical cyclones (TCs) on different spatial/temporal-scale environmental fields is to contrast the original circulation with the circulation from which the TCs have been removed. Although dynamical balance is required for analyzing TC contributions, the dynamic balance of TC-removed fields obtained by the existing TC removal method (which is widely used in the TC bogus procedure) is often ignored. In this paper, a TC removal method incorporating the potential vorticity (PV) inversion technique is proposed and its application to climate study is demonstrated. This method objectively detects the TCs' positive PV disturbance, which is strong with a deep structure and overwhelmingly dominates the relatively weak and thin negative PV disturbance. The TC-removed field is well-balanced due to the dynamic balance consideration in the PV inversion framework. This approach isolates TC vortices, which are stronger and have a wider range of impacts compared with the TC components derived by the existing removal methods. The TC-removed fields obtained by the existing and the proposed methods are profoundly different, especially in dynamic balance. The TC contribution to intraseasonal variance and seasonal mean circulation in the tropical western North Pacific is examined. The existence of TCs enhances the amplitude and propagation of intraseasonal oscillation and strengthens the seasonal mean circulations such as the low-level monsoon trough and upper-level anticyclone in the region. Whereas the existing and the proposed TC removal techniques yield consistent results, the proposed technique yields larger TC contributions to the seasonal-mean circulation and the amplitude and northward propagation tendency of intraseasonal oscillation.
\end{abstract}

\section{Introduction}

Several studies have revealed the large-scale environmental effects on a tropical cyclone (TC) during its life cycle: genesis (Gray 1998; Ritchie and Holland 1999), development (DeMaria 1996; Ritchie and Frank 2007; Hendricks et al. 2010), and movement (Franklin et al. 1996; Sobel and Camargo 2005; Galarneau and Davis 2013). By contrast, few studies have considered the effects exerted by TCs on the large-scale environment. By comparing original reanalysis data with TC-removed data, Hsu et al. (2008a,b) demonstrated that TCs contribute more than $50 \%$ to intraseasonal

Sho Arakane

shokun5656@gmail.com

1 Research Center for Environmental Changes, Academia Sinica, Taipei 115, Taiwan

2 Present Address: Atmosphere and Ocean Research Institute, The University of Tokyo, Kashiwa 277-8568, Japan variance (ISV) of 850 -hPa vorticity along TC tracks in the tropical western North Pacific (WNP) during boreal summers when TC and intraseasonal oscillation (ISO) activities are strong. Ko et al. (2012) also used the same method and demonstrated that TCs enhance the amplitudes of vorticity and kinetic energy of the TC/submonthly wave pattern in the WNP by more than 50\%. The TC removal technique proposed by Kurihara et al. (1995) [hereinafter called Kurihara filtering (KF)] was employed in the aforementioned studies. Their procedure is described briefly as follows. An 850-hPa wind field was divided into basic and disturbance fields, and its TC center and domain were then determined using tangential wind profile of the disturbance field. For all atmospheric variables, the disturbance field within the TC domain was removed and replaced with a smoothed field.

KF was widely used in the TC numerical experiments (Cheung and Chan 2001; Wu et al. 2002; Kwon et al. 2002; Rogers et al. 2003). Ross and Kurihara (1995) used KF to create an initial field of sensitivity experiment in which a TC was removed. Moreover, in dynamical initialization scheme for 
Table 1 Tunable parameters for determining the TC removal domain used in Kurihara et al. (1995) and Galarneau and Davis (2013)

\begin{tabular}{|c|c|c|}
\hline Filtering method & Tunable parameter & Value used in the original study (reference in the original study) \\
\hline \multirow[t]{5}{*}{ KF } & Search direction $(\theta)$ for wind profile test & 24 radial directions (every $15^{\circ}$ angle) (Sect. 2.3 ) \\
\hline & $\begin{array}{l}\text { Parameters to determine the range of a start- } \\
\text { ing point for wind profile test }(a, b)\end{array}$ & $a=0.5, \mathrm{~b}=0.75($ Appendix A) \\
\hline & $\begin{array}{l}\text { Condition to determine an appropriate starting } \\
\text { radius for each direction }\left[r_{f}(\theta)\right]\end{array}$ & $\begin{array}{l}v_{\tan }<6 \mathrm{~m} \mathrm{~s}^{-1} \text { and }-\partial v_{\tan } / \partial r<4 \times 10^{-6} \mathrm{~s}^{-1} \text { (the second } r \text { for which } \\
\text { this is true), or } v_{\tan }<3 \mathrm{~m} \mathrm{~s}^{-1}[r: \text { the distance from the TC center, } \\
v_{\tan }(r, \theta): \text { the tangential component of the disturbance wind centered } \\
\text { on the TC center] (Sect. 2.3) }\end{array}$ \\
\hline & Maximum $r_{f}(\theta)$ & 1200 km (Sect. 2.3) \\
\hline & Definition of TC domain radius $\left[r_{0}(\theta)\right]$ & $r_{0}(\theta)=1.25 r_{f}(\theta)($ Sect. 2.3) \\
\hline GDF & TC domain radius $\left(r_{0}\right)$ & $\begin{array}{l}\text { Depend on each TC } \\
\text { (Sect. } 2.2 \text { and Figs. 10,11) }\end{array}$ \\
\hline
\end{tabular}

TC simulations, Cha and Wang (2013) and Wang et al. (2013) employed KF to separate the TC vortex component and the environmental component. To calculate the large-scale vertical wind shear, Wang et al. (2015) used the filtered basic wind field obtained by KF.

The employment of this type of TC removal technique is a powerful way to investigate the effects of a TC on the largescale environment. However, the use of KF results has some problems. First, although a TC can sometimes tilt vertically, the center and domain are fixed at all layers because they are determined by the 850-hPa wind field only. Second, the dynamical balance is not considered in both the TC-removed component and the remaining circulation. The consideration of the dynamical balance in the TC removal fields is important when other dynamical fields, e.g., vertical velocity, temperature, and geopotential fields, are analyzed to obtain a complete structure associated with TC. These inherent problems in KF are partially dealt with in the new TC removal technique proposed by Winterbottom and Chassignet (2011); the TC center and domain are determined individually at each level. The domains for the geopotential height and temperature fields are 1.25 times of the domain for the wind field, considering the larger influence of TC on these fields compared to the winds. However, the balance between variables was not considered in the removal process, either. In the numerical simulations, the dynamical imbalance in the TC-removed fields is often overcome through dynamical initialization.

Galarneau and Davis (2013) proposed a new decomposition technique; the study decomposed wind fields into TC and non-TC wind components to investigate TCs' steering flows [hereinafter called Galarneau and Davis filtering (GDF)]. The TC components of streamfunction $(\Psi)$ and velocity potential $(\chi)$ are defined as:

$\nabla^{2} \Psi=\left\{\begin{array}{l}\zeta,\left(r \leq r_{0}\right) \\ 0,\left(r>r_{0}\right)\end{array}\right.$ and $\nabla^{2} \chi=\left\{\begin{array}{l}\delta,\left(r \leq r_{0}\right) \\ 0,\left(r>r_{0}\right)\end{array}\right.$,

where $\zeta$ is the relative vorticity, $\delta$ is the divergence, $\nabla$ is the two-dimensional gradient operator, and $r$ and $r_{0}$ are the distance from the TC center and the radius of TC domain, respectively. Using these Helmholtz equations, the TC-associated rotational and divergent wind components are then calculated by the obtained TC-associated streamfunction and velocity potential, respectively. GDF removes all of rotational and divergent wind fields inside the pre-determined $\mathrm{TC}$ domain, unlike KF that determines and retains the nonTC background circulation, and does not decompose other variables such as geopotential and temperature. Similar vortex removal method is used for the vortex removal scheme in the Weather Research and Forecasting (WRF) model. In the scheme, using the nondivergent wind, the balance equation is solved to obtain geopotential, and then temperature is computed in the vortex-removed field.

Here, we compare and summarize the existing TC removal methods, $\mathrm{KF}$ and GDF. In KF, the TC domain is determined by using 850 -hPa radial disturbance tangential wind profile from the TC center. In this procedure, 24 radial directions from the TC center are tested; the obtained removal domain is generally not circular. The TC center and domain remain fixed vertically at all levels. The wind, geopotential, and temperature associated with the target TC are then individually removed within the domain without considering the balance among the variables. On the other hand, GDF's TC-removal domains are circular: all vorticity and divergence are removed within the circular domain whose extent depends on the TC considered. Then, the rotational and the divergent wind fields after the TC removal are recovered by solving the Helmholtz equation. The tunable parameters to detect the removal domains used in these methods are listed in Table 1. To obtain the TC removal domain, several tunable parameters [e.g., (a, b), $r_{f}(\theta)$, and $r_{0}(\theta)$ ] are used in KF. By contrast, only one tunable parameter $\left(r_{0}\right)$ is used in GDF.

In view of the dynamical imbalance issue in the existing TC-removal schemes, this paper proposes a TC removal technique in which potential vorticity (PV) inversion (PVI; Davis and Emanuel 1991; Wang and Zhang 2003) 
is employed. The proposed removal technique is applied to reanalysis data during June-October (JJASO) 2004. The derived fields are dynamically balanced in the PVI framework. We compare the TC-removed fields derived using the traditional and the proposed TC removal techniques, and reexamine the TC contribution to intraseasonal variance and seasonal-mean circulation in the tropical WNP discussed in Hsu et al. (2008a) with dynamically well-balanced TCremoved data. This paper is organized as follows: Sect. 2 describes the data and the procedure of this algorithm. Section 3 compares the TC-removed field with the existing and the proposed methods. The influences of TC on the intraseasonal variance of vorticity and on the seasonal-mean geopotential height and temperature fields are discussed in Sect. 4. Finally, in Sect. 5, we conclude this study and discuss the advantages of the proposed TC removal technique for climate study by investigating the TC effect on the largescale environment.

\section{Methodology}

\subsection{Data}

The atmospheric variables used in this study were geopotential, horizontal and vertical winds, temperature, and specific humidity; the data were taken from the Japanese 55-year Reanalysis Project (JRA-55; Kobayashi et al. 2015; Harada et al. 2016) provided by the Japan Meteorological Agency. The JRA-55 is a $6-\mathrm{h} 1.25^{\circ}$ latitude/longitude gridded data set. This study analyzes 5 months of data from JJASO 2004. An advantage of JRA-55 is that the data set assimilates tropical cyclone wind retrievals (TCRs) derived from best track data. Because of the TCRs, the intensity and center location of TCs are better represented in JRA-55 compared with other reanalysis data (Murakami 2014).

To decompose the PV anomalies (PVAs) associated with TCs, the best track data provided by the Regional Specialized Meteorological Center, Tokyo (RSMC-Tokyo) are used. TCs in the categories of tropical storm, severe tropical storm (STS), and typhoon (TY) during JJASO 2004 in the RSMCTokyo best track data are defined as analysis objects in this study. The use of the RSMC-Tokyo best track data for the decomposition is discussed later.

\subsection{PV inversion}

\subsubsection{Total PV inversion}

The PVI technique used in this study was developed by Davis and Emanuel (1991). Assuming that the irrotational and vertical winds are negligible in the divergence equation, the balance equation is derived as $\nabla^{2} \boldsymbol{\Phi}=\nabla \cdot(f \nabla \Psi)+2\left[\frac{\partial^{2} \Psi}{\partial x^{2}} \frac{\partial^{2} \Psi}{\partial y^{2}}-\left(\frac{\partial^{2} \Psi}{\partial x \partial y}\right)^{2}\right]$

where $\Phi$ is geopotential and $f$ is the Coriolis parameter. Because this study is focused on TC located at low latitudes where water vapor is rich, the form of PV should consider the moisture effect as demonstrated by Wang and Zhang (2003). The approximate definition of PV that includes the moisture effect in $\pi\left[=C_{p}\left(p / p_{0}\right)^{\kappa}\right]$ coordinate is written as

$q=-\frac{g \kappa \pi}{p}\left(\eta \frac{\partial \theta_{v}}{\partial \pi}-\frac{\partial v}{\partial \pi} \frac{\partial \theta_{v}}{\partial x}+\frac{\partial u}{\partial \pi} \frac{\partial \theta_{v}}{\partial y}\right)$,

where $\kappa=R_{d} / C_{p}$ is the ratio of gas constant for dry air $\left(R_{d}\right)$ and specific heat at constant pressure $\left(C_{p}\right), p$ is pressure, $p_{0}$ is reference pressure $(=1000 \mathrm{hPa}), g$ is gravitational acceleration, $u$ is eastward wind speed, $v$ is northward wind speed, $\eta$ is the vertical component of absolute vorticity, $\theta_{v}$ is virtual potential temperature $[=(1+0.608 s) \theta$, where $s$ is specific humidity and $\theta$ is potential temperature], and $q$ is the PV. In Eq. (2), the terms that include vertical velocity are neglected. Under the assumptions of hydrostatic balance, $\theta_{v}=-\partial \Phi / \partial \pi$, and that the nondivergent wind is much greater than the irrotational wind, Eq. (2) can be rewritten as

$q=-\frac{g \kappa \pi}{p}\left[\left(f+\nabla^{2} \Psi\right) \frac{\partial^{2} \Phi}{\partial \pi^{2}}-\frac{\partial^{2} \Psi}{\partial x \partial \pi} \frac{\partial^{2} \Phi}{\partial x \partial \pi}-\frac{\partial^{2} \Psi}{\partial y \partial \pi} \frac{\partial^{2} \Phi}{\partial y \partial \pi}\right]$.

Equations (1) and (3) have two unknowns. We can calculate them using the method in Davis and Emanuel (1991) and Wang and Zhang (2003) with the $q$ distribution, $\theta_{v}$ on the upper and bottom boundaries, and $\Phi$ and $\Psi$ on the lateral boundaries.

The domain used for PVI extends from $0^{\circ}$ to $60^{\circ} \mathrm{N}$ and from $90^{\circ} \mathrm{E}$ to $150^{\circ} \mathrm{W}$ with a horizontal resolution of $1.25^{\circ}$ latitude/longitude. The solution occasionally includes an unstable layer because of numerical error induced by an inhomogeneous vertical grid interval in the $\pi$ coordinate. To prevent the appearance of the unstable layer in the solution, the data on the inhomogeneous vertical grid is reconstructed into a homogeneous one through cubic spline interpolation. The reconstructed data has 26 layers, the level at $k=1(26)$ of the reconstructed data satisfies $\pi=C_{p}\left(0.5 C_{p}\right)$, and the constant vertical grid interval is $\Delta \pi=0.02 C_{p}$.

The $\theta_{v}$ fields at the levels of $\pi=0.99 C_{p}$ and $0.51 C_{p}$ (approximately 965 and $95 \mathrm{hPa}$ ) are used for the upper and bottom boundary conditions that satisfy $\theta_{v}=-\partial \Phi / \partial \pi=-f_{0} \partial \Psi / \partial \pi$, where $f_{0}$ is the Coriolis parameter at $30^{\circ} \mathrm{N}$. The Dirichlet boundary condition (fixed $\Phi$ and $\Psi$ ) is used for the lateral boundary condition. We attempted to use the Neumann boundary condition for solving the system; however, a stable solution is not always obtainable. Wu and Emanuel (1995) argued that the reason is probably the imbalance between mass and wind fields near the equator. To overcome this problem, they considered $10^{\circ}$ or $12.5^{\circ}$ 
$\mathrm{N}$ as the southern boundary of the inversion domain. This study requires the inverted geopotential and streamfunction at low latitudes. Therefore, we select the Dirichlet boundary condition to stabilize the solution.

The PVI is executed for 612 (every $6 \mathrm{~h}$ for 5 months) cases in total. The solution diverges in some cases and tends to converge when a smaller under-relaxation parameter for $\Psi$ (see Davis and Emanuel 1991) is applied; however, the use of the smaller parameter requires more computation time. To overcome this solution problem, the parameter becomes smaller automatically in the code when the solution diverges, and then the calculation is executed again. No smoothing for any variable is required in our PVI code because of the adoption of parameter correction and the application of the parameter $\varepsilon$ introduced by Wang and Zhang (2003), which prevents the nonellipticity problem for the 3-D Poisson equation (for more details, see Wang and Zhang 2003).

\subsubsection{Piecewise PV inversion}

To recover the balanced mass and circulation fields associated with PV perturbations of TCs, piecewise PV inversion (PPVI) is employed. The method of Davis and Emanuel (1991) is used for constructing the PPVI system. First, we define $\bar{\Phi}$ and $\bar{\Psi}$, which satisfy Eqs. (1) and (3) for a given $\bar{q}$ :

$\nabla^{2} \overline{\boldsymbol{\Phi}}=\nabla \cdot(f \nabla \bar{\Psi})+2\left[\frac{\partial^{2} \bar{\Psi}}{\partial x^{2}} \frac{\partial^{2} \bar{\Psi}}{\partial y^{2}}-\left(\frac{\partial^{2} \bar{\Psi}}{\partial x \partial y}\right)^{2}\right]$,

$\bar{q}=-\frac{g \kappa \pi}{p}\left[\left(f+\nabla^{2} \bar{\Psi}\right) \frac{\partial^{2} \bar{\Phi}}{\partial \pi^{2}}-\frac{\partial^{2} \bar{\Psi}}{\partial x \partial \pi} \frac{\partial^{2} \bar{\Phi}}{\partial x \partial \pi}-\frac{\partial^{2} \bar{\Psi}}{\partial y \partial \pi} \frac{\partial^{2} \bar{\Phi}}{\partial y \partial \pi}\right]$,

where $q$ is an arbitrary averaged PV. By substituting $\Phi=\bar{\Phi}+\Phi^{\prime}, \Psi=\bar{\Psi}+\Psi^{\prime}$, and $q=\bar{q}+q^{\prime}$ into Eqs. (1) and (3), and by using Eqs. (4) and (5), the PPVI system can be derived as

$\nabla^{2} \Phi^{\prime}=\nabla \cdot\left(f \nabla \Psi^{\prime}\right)+2\left[\frac{\partial^{2} \Psi^{*}}{\partial x^{2}} \frac{\partial^{2} \Psi^{\prime}}{\partial y^{2}}+\frac{\partial^{2} \Psi^{\prime}}{\partial x^{2}} \frac{\partial^{2} \Psi^{*}}{\partial y^{2}}-2 \frac{\partial^{2} \Psi^{*}}{\partial x \partial y} \frac{\partial^{2} \Psi^{\prime}}{\partial x \partial y}\right]$,

$$
\begin{aligned}
q^{\prime}= & -\frac{g \kappa \pi}{p}\left[\left(f+\nabla^{2} \Psi^{*}\right) \frac{\partial^{2} \Phi^{\prime}}{\partial \pi^{2}}+\frac{\partial^{2} \Phi^{*}}{\partial \pi^{2}} \nabla^{2} \Psi^{\prime}\right. \\
& \left.-\frac{\partial^{2} \Psi^{*}}{\partial x \partial \pi} \frac{\partial^{2} \Phi^{\prime}}{\partial x \partial \pi}-\frac{\partial^{2} \Phi^{*}}{\partial x \partial \pi} \frac{\partial^{2} \Psi^{\prime}}{\partial x \partial \pi}-\frac{\partial^{2} \Psi^{*}}{\partial y \partial \pi} \frac{\partial^{2} \Phi^{\prime}}{\partial y \partial \pi}-\frac{\partial^{2} \Phi^{*}}{\partial y \partial \pi} \frac{\partial^{2} \Psi^{\prime}}{\partial y \partial \pi}\right],
\end{aligned}
$$

where []$\left.\left.^{*}=\overline{[}\right]+1 / 2[]^{\prime}=1 / 2([]+\overline{[}]\right)$.

Given the PVA associated with TC $\left(q^{\prime}\right)$, TC-associated geopotential $\left(\Phi^{\prime}\right)$ and streamfunction $\left(\Psi^{\prime}\right)$ are solved in the PPVI system. The distributions of $\Phi^{\prime}$ and $\Psi^{\prime}$ depend on the definition of $q^{\prime}(=q-\bar{q})$, which in turn depends on how the averaged PV is defined. That is, the derived $\Phi^{\prime}$ and $\Psi^{\prime}$ depend on the definition of $\bar{q}$. Therefore, care must be exercised when handling the arbitrary averaging of PV as discussed below.

\subsection{PV partitioning}

\subsubsection{Basic framework of decomposition}

The PVAs generated by TCs are mainly characterized by the following two features: (1) the positive PVA concentrated on the TC center in the troposphere, and (2) the negative PVA widely spread over the TC in the upper troposphere and the lower stratosphere (Fig. 1). As discussed in Haynes and McIntyre (1987), the PV associated with a TC should follow the volume integral constraint of PV: the integral of PV in a certain volume bounded by two isentropic surfaces is constant. That is, a positive PV produced by diabatic heating must be compensated by an equal and negative PV in the volume. This constraint should be taken into account in principle when the TC-associated PVAs are isolated from the large-scale environmental PV. Whether such a constraint can be obtained in real data is explored below.

The characteristics of TC-associated positive PVAs are clearly observed in the cases of TY Mindulle and STS Tingting (Fig. 1a) and STS Ma-on (Fig. 1d): these positive PVA are spatially isolated and exist in the troposphere. On the other hand, the distributions of the negative PVAs associated with the TCs are complicated. In the case of TY Mindulle and STS Tingting, a negative PVA encircles each positive PVA (Fig. 1a-c). These encircling negative PVAs are presumably associated with their respective TC. However, in the case of STS Ma-on, the distribution of negative PVA in the upper troposphere is highly asymmetric and spreads out in an area much larger than the TC itself; the negative PVA is associated with an elongated band (i.e., a trough) to the north of STS Ma-on (Fig. 1d-f). Such asymmetric and ill-defined TC-related negative PVAs are observed in most of TC cases. By contrast, a clear negative PVA encircling the center of TC such as in Fig. 1a-c is rarely observed. The horizontal distributions of the asymmetric negative PVAs observed in most of TC cases are presumably associated with the large-scale wind fields in the upper troposphere. Greater asymmetry of the negative PVA in the upper troposphere tends to be observed when TCs move into the midlatitudes (not shown). Moreover, as shown in Fig. 1a, d, the negative PVAs over the TCs extend continuously from the upper troposphere to the lower stratosphere. The upper boundaries of the negative PVAs associated with the TCs cannot be well defined.

In contrast to the partitioning of the positive PVA associated with the TC, it is more difficult to partition the asymmetric negative PVA into its TC and non-TC components. A clean way for separating TC and non-TC negative PVAs is yet to be identified. For this reason, we identify an isolated 
TY Mindulle and STS Tingting at 00Z28JUN2004

(a) Vert. Cross Section

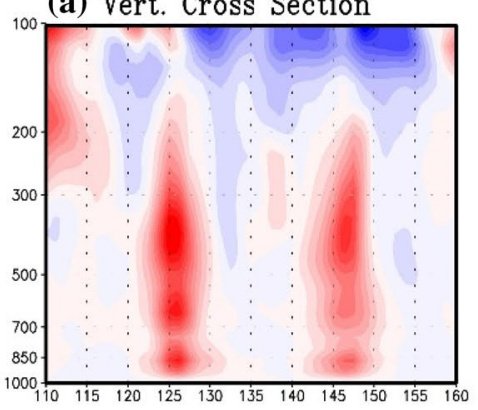

STS Ma-on at 00Z060CT2004

(d) Vert. Cross Section

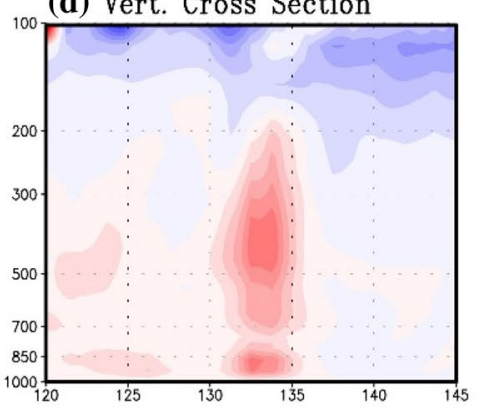

(b) $209.7 \mathrm{hPa}$

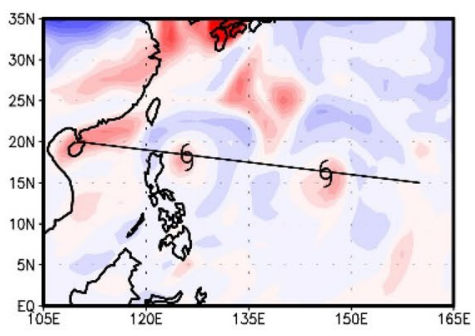

(e) $209.7 \mathrm{hPa}$

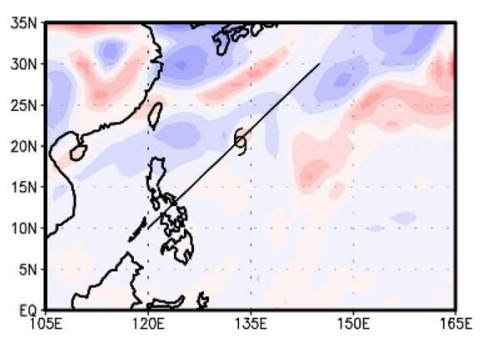

(c) $148.6 \mathrm{hPa}$

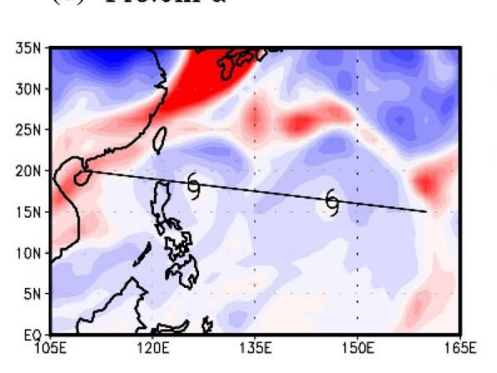

(f) $148.6 \mathrm{hPa}$

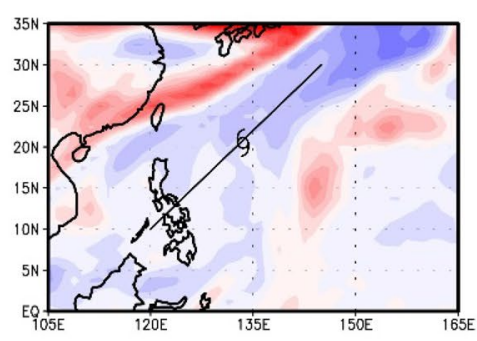

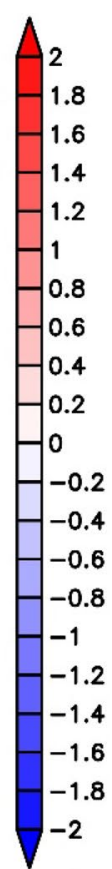

(PVU)
Fig. 1 (Top) Distributions of PV disturbance (in PVU). a Vertical cross section along the line indicated in the following plan views; $\mathbf{b}$ plan view at $209.7 \mathrm{hPa}$; and c plan view at $148.6 \mathrm{hPa}$ for the case of Typhoon Mindulle (at $18.1^{\circ} \mathrm{N}, 126.0^{\circ} \mathrm{E}$, MSLP: $950 \mathrm{hPa}$ ) and Severe
Tropical Storm Tingting (at $16.1^{\circ} \mathrm{N}, 146.3^{\circ} \mathrm{E}$, MSLP: $980 \mathrm{hPa}$ ) at 0000 UTC on June 28. (Bottom) Same as the top panel but for the case of Severe Tropical Storm Ma-on (at $20.5^{\circ} \mathrm{N}, 133.5^{\circ} \mathrm{E}$, MSLP: $985 \mathrm{hPa}$ ) at $0000 \mathrm{UTC}$ on October 6 positive PVA in the troposphere within TC domain as proposed above and negative PVAs within the $800-\mathrm{km}$ radius from the TC center, reported in the best-track data, from the surface to the top.

Isolating the TC circulation would involve a definition of a mean (environmental) field, which depends on the purpose of the study. For example, Davis and Emanuel (1991) defined the mean as a 5-day time-averaged field in the extratropical cyclone study. Although $\mathrm{Wu}$ and Emanuel (1995) also employed time averaging in their study of TC movement, the averaging period was 3 months. The mean geopotential and streamfunction fields in these studies are solved in the system of Eqs. (4) and (5). On the other hand, spatially averaged fields were used for the mean fields in other studies. Focusing on a highly axisymmetric structure of TC, an axisymmetric averaged streamfunction circling the TC center is adopted as a mean streamfunction field, and mean geopotential and PV fields are solved by Eqs. (4) and (5), respectively (Shapiro 1996; Wu et al. 2003, 2004). Wang and Zhang (2003) used the axisymmetric averaging for geopotential fields to avoid the appearance of negative static stability near the top of the planetary boundary layer; mean streamfunction and PV fields were then obtained from the gradient wind balance relation and Eq. (5), respectively.
Although the time-averaged fields can be easily calculated, the shortcoming of time averaging for TC study is that the obtained mean field is severely influenced by the presence of TCs. By contrast, the advantage of using the axisymmetric averaging is that the TC's axisymmetric structure can be completely removed when the TC center is clearly defined. However, TC sometimes tilts vertically, and thus, it becomes complicated to define the TC center. In this situation, an employment of axisymmetric averaging may cause errors in the mean and perturbation fields.

In this study, a new decomposition method in which both time and spatial averaging are applied is adopted to obtain the mean PV field, which is not related to the presence of TC. We first decompose PV at a certain time such that

$q(t)=\overline{q(t)}^{t}+q^{\prime}(t)$

where $\overline{[}^{t}$ represents a 31 -day time-averaging field, ${ }^{1}$ called the basic field, and $q^{\prime}(t)$ is the perturbation PV, which is the deviation from the basic PV field. Then, $q^{\prime}(t)$ is separated into a TC and a non-TC component (the separating method is described in the following subsection):

\footnotetext{
1 The application of different time mean periods have negligible impacts on the results (not shown).
} 
Fig. 2 Flowchart illustrating the procedure of creating the mean and TC component fields (see the text). The fields in italic are used for the analysis

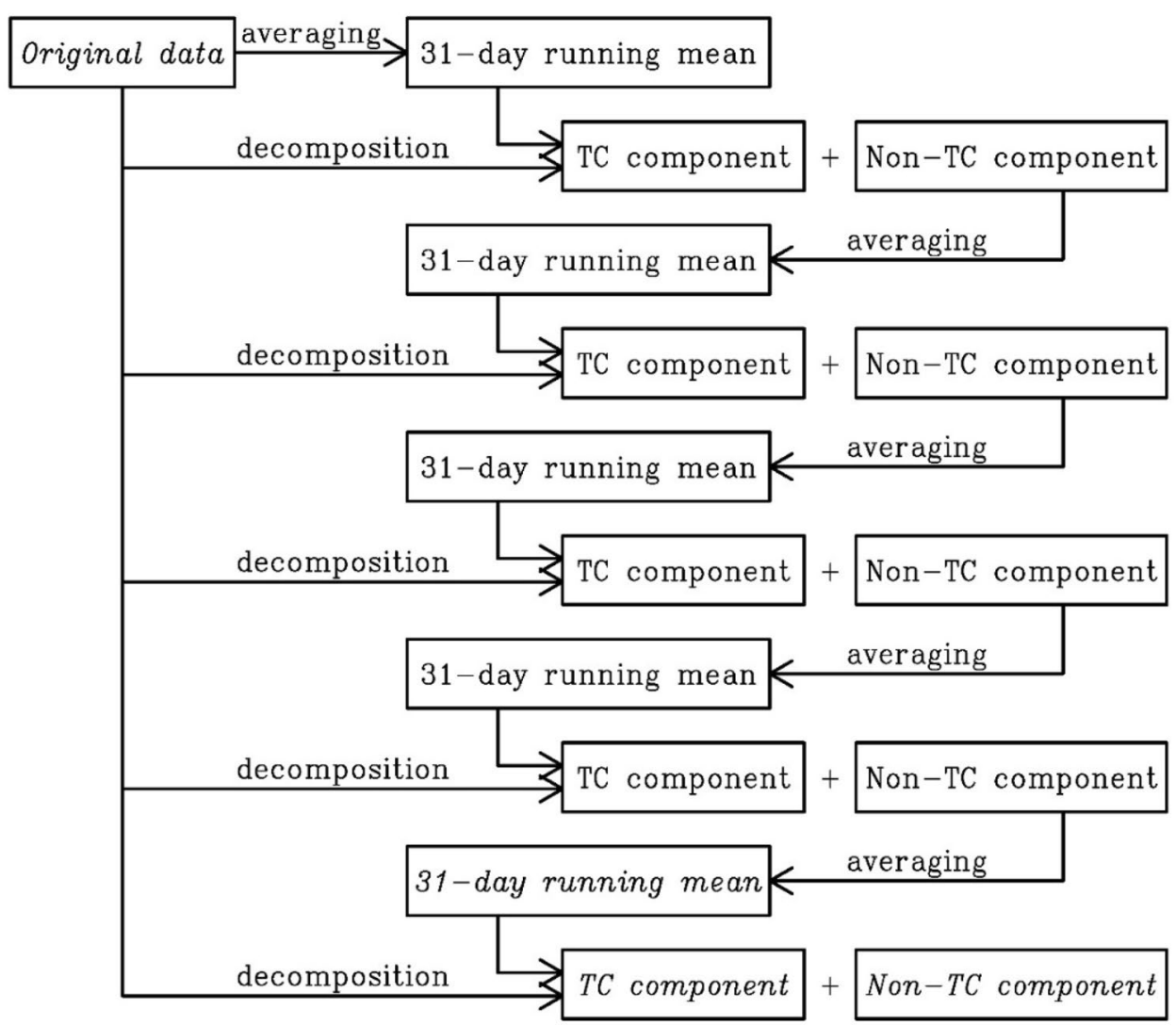

$q^{\prime}(t)=q_{\mathrm{TC}}(t)+q_{\mathrm{noTC}}(t)$.

Finally, the environmental PV field is obtained by combining the non-TC part with the basic PV field:

$q_{\mathrm{E}}(t)=\overline{q(t)}^{t}+q_{\mathrm{noTC}}(t)$.

Equation (8) can be written as

$q(t)=q_{\mathrm{TC}}(t)+q_{\mathrm{E}}(t)$.

This is the same as Kurihara et al. (1995), except for the calculation of the basic field; in their study, the basic field was defined by spatial filtering method. As stated above, $\overline{q(t)}^{t}$ includes the effect of the presence of TC. To cleanly remove the imprints of TC in the basic PV field, we further recalculate the basic PV field using the obtained $q_{\mathrm{E}}(t)$, and separate the TC and non-TC components again from the new perturbation field, $q(t)-{\overline{q_{\mathrm{E}}(t)}}^{t}$ :

$q(t)={\overline{q_{\mathrm{E}}(t)}}^{t}+\left.q_{\mathrm{TC}}(t)\right|_{\text {new }}+\left.\left.q_{\text {noTC }}(t)\right|_{\text {new }} \equiv q_{\mathrm{E}}(t)\right|_{\text {new }}+\left.q_{\mathrm{TC}}(t)\right|_{\text {new }}$.

The TC imprints in the new environmental PV field, $\left.q_{\mathrm{E}}(t)\right|_{\text {new }}$ are reduced than those in the previous environmental PV field $q_{\mathrm{E}}(t)$. However, the obtained new environmental PV field still has weaker TC imprints; therefore, we repeat this procedure. That is,

$$
\begin{aligned}
q(t) & =\overline{q(t)}^{t}+q_{\mathrm{TC}}^{1}(t)+q_{\mathrm{noTC}}^{1}(t) \equiv q_{\mathrm{E}}^{1}(t)+q_{\mathrm{TC}}^{1}(t), \\
& ={\overline{q_{\mathrm{E}}^{1}(t)}}^{t}+q_{\mathrm{TC}}^{2}(t)+q_{\mathrm{noTC}}^{2}(t) \equiv q_{\mathrm{E}}^{2}(t)+q_{\mathrm{TC}}^{2}(t), \\
& ={\overline{q_{\mathrm{E}}^{2}(t)}}^{t}+q_{\mathrm{TC}}^{3}(t)+q_{\mathrm{noTC}}^{3}(t) \equiv q_{\mathrm{E}}^{3}(t)+q_{\mathrm{TC}}^{3}(t), \\
& \vdots \\
q(t) & ={\overline{q_{\mathrm{E}}^{n-1}(t)}}^{t}+q_{\mathrm{TC}}^{n}(t)+q_{\mathrm{noTC}}^{n}(t) \equiv q_{\mathrm{E}}^{n}(t)+q_{\mathrm{TC}}^{n}(t),
\end{aligned}
$$

where the superscript $n$ denotes the number of iterations. The TC effect on the environmental field disappears gradually with the iterations.

This decomposition procedure is summarized in Fig. 2 and the obtained basic (time-averaged) fields after each iteration at 0000 UTC 16 August 2004 are shown in Fig. 3. After the first iteration, there exist two PV maxima, which are associated with the TCs, near $25^{\circ} \mathrm{N}, 125^{\circ} \mathrm{E}$ and $27^{\circ} \mathrm{N}, 133^{\circ}$ E (Fig. 3a). The amplitudes of these maxima decrease after the second iteration (Fig. 3b). These PV extrema are virtually removed after the 4th and 5th iterations, and the distributions of ${\overline{q_{\mathrm{E}}^{3}(t)}}^{t}$ and ${\overline{q_{\mathrm{E}}^{4}(t)}}^{t}$ are almost identical (Fig. 3c, d). Thus, the TC imprints are effectively removed in ${\overline{q_{\mathrm{E}}^{4}(t)}}^{t}$ (the iteration is repeated five times for this study), and the usage of this time mean field with little trace of the TC imprints is valid for the purpose of this study. Another important feature of the basic field is that the PV value in a TC-free region is 
PV (31-day mean), 691.6hPa, 0000 UTC August 16, 2004
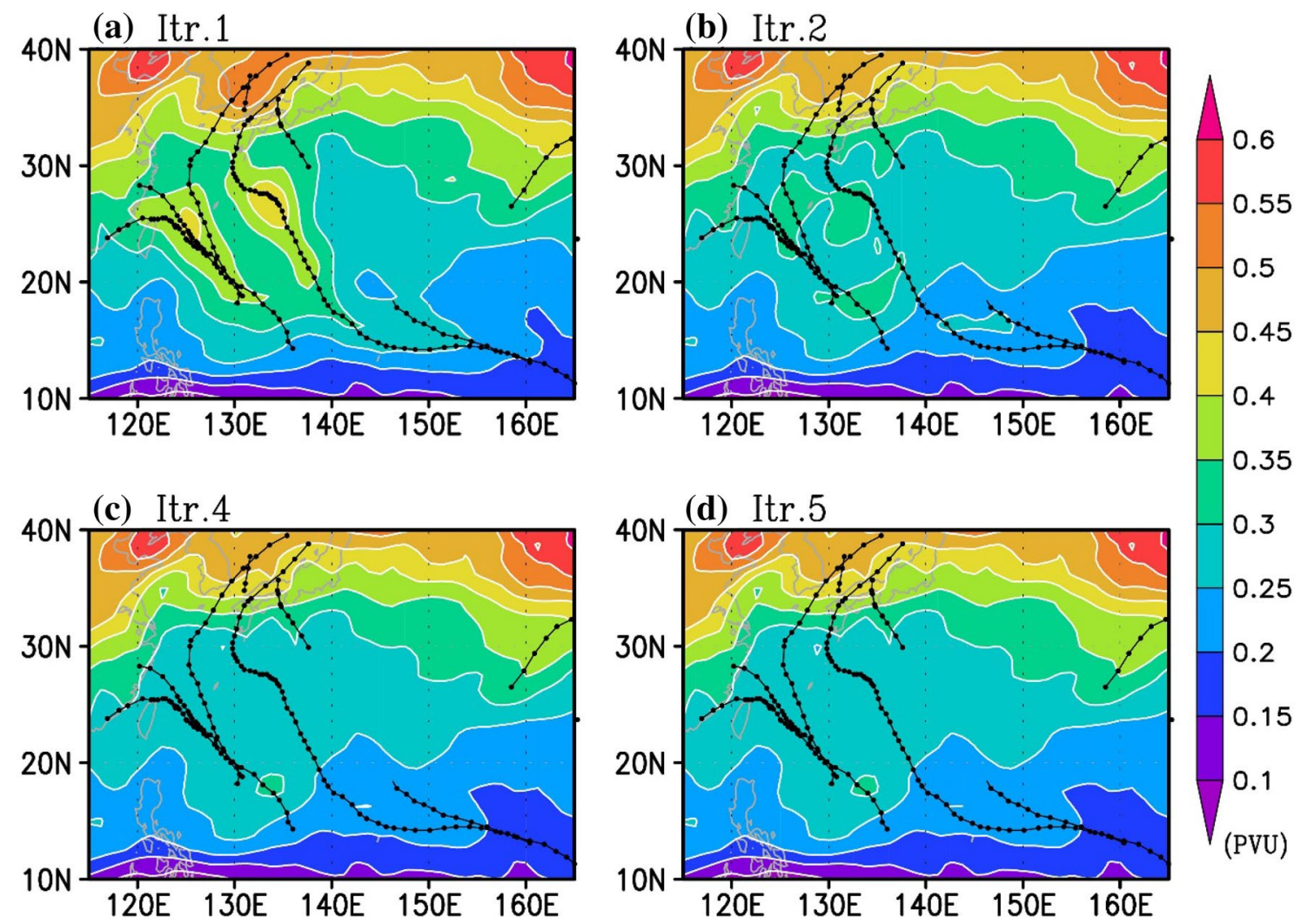

\section{.3 \\ .25 \\ .15 \\ 1}

0.2

PVU)

Fig. 3. Basic (31-day running mean) PV fields at 0000 UTC on August 16, 2004 at $691.6 \mathrm{hPa}$ after 1, 2, 4, and 5 iterations: a $\overline{q(t)}^{t}$; b ${\overline{q_{E}^{1}(t)}}^{t}$; c $q_{E}^{3}(t)$; and $\mathbf{d} q_{E}^{4}(t)$ (see the text). Dots indicate the locations of TCs that exist during the time-averaging period

unchanged by the iterative method because of the definition of the environmental component of PV. In addition, note that the basic field varies with time step because it is a 31-day running mean.

\subsubsection{Determination of TC domain for positive PVA}

The basic procedure for the determination of $\mathrm{TC}$ domain for positive PVA in this study follows that proposed by Kurihara et al. (1995) and Kwon and Cheong (2010). The procedure is as follows: the TC center at each level and TC height are detected first, and then the domain boundary of the TC in 360 directions from the TC center is determined, as described in the next paragraph. After the TC domain detection, tropospheric positive PVA is extracted for separating the TC and non-TC components (see previous subsection).

In Kurihara et al. (1995) and Kwon and Cheong (2010), the bilinearly interpolated disturbance field at $850 \mathrm{hPa}$ was used to determine the TC center and domain. However, using one layer for defining the TC center (and domain) may lead to errors when the TC structure tilts in the vertical.
Therefore, we define the TC center and domain individually at each layer. Here, the TC center is defined as the location of maximum vorticity averaged within a $250-\mathrm{km}$ disk. The precision of the TC center is $(1.25 / 16)^{\circ}$; thus, the TC center is typically not located on a grid point. The TC position recorded in the RSMC-Tokyo best track data is used for an initial guess first, and then the TC center at the level $k=2$ is determined. PVAs associated with TCs at $k=1$ do not need to be determined because the PPVI system does not require PVAs at the lowest level. It is the potential temperature anomaly that PPVI system needs at the lowest level. The anomaly within a distance of $1200 \mathrm{~km}$ from the TC position recorded in the RSMC-Tokyo best track data is defined as the TC-associated potential temperature anomaly. After the determination of TC center at $k=2$, a location of TC center at $k=3$ is determined using the information of the center location at $k=2$; this procedure is repeated continuously upward. However, this procedure may pick up another meteorological system that has strong vorticity in the vicinity of the TC. To avoid this potential problem, the vertically connected TC center, so-called the vortex axis, is examined; the TC center detection repeated upwardly is terminated when 


\section{PV(TC), 691.6hPa}

(a) Tropical Storm Megi

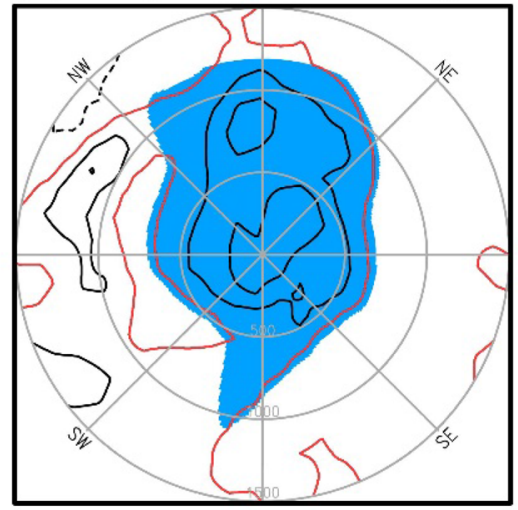

(b)

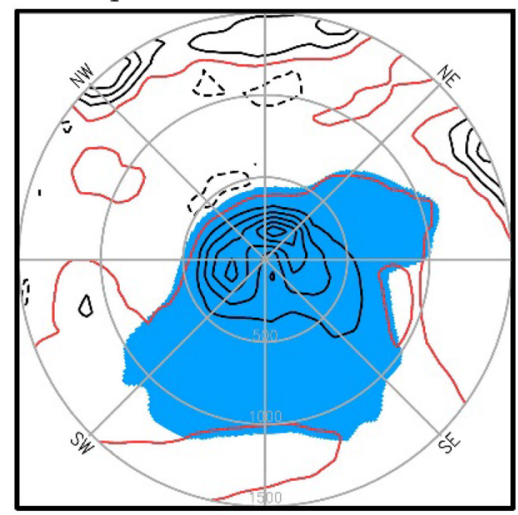

(c) Typhoon Chaba

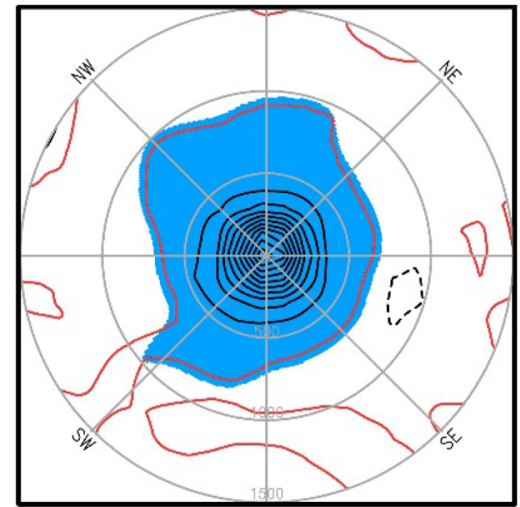

Fig. 4 Distributions of the PV disturbance at $691.6 \mathrm{hPa}$ : a Tropical Storm Megi at 1200 UTC 16 (MSLP: $992 \mathrm{hPa}$ ); b Tropical Storm Rananim at 1800 UTC 12 (MSLP: $975 \mathrm{hPa}$ ); and c Typhoon Chaba at 1800 UTC 12 (MSLP: $910 \mathrm{hPa}$ ) August 2004. The contour interval is

the vertical gradient of the vortex axis exceeds the threshold of $300 / \Delta \pi\left[\mathrm{km} \mathrm{J}^{-1} \mathrm{~kg} \mathrm{~K}\right]$. This threshold is empirically chosen and corresponds approximately to a two-grid distance per one layer at $1.25^{\circ}$ horizontal resolution.

After determining the TC center, by examining the PV disturbance ${ }^{2}$ profile along 360 radial directions, the extent of the TC domain is determined at all layers. Going outward from a point $200 \mathrm{~km}$ away from the obtained TC center, each profile is tested at radial intervals of $10 \mathrm{~km}$. It means that the profile from 0 to $200 \mathrm{~km}$ is not tested. The reason will be discussed later in this paragraph. The domain boundary at each angle is defined as the point where $q_{\text {test }}<0.05$ PVU (PV unit: $10^{-6} \mathrm{~m}^{2} \mathrm{~kg}^{-1} \mathrm{~s}^{-1} \mathrm{~K}$ ) is first satisfied, where $q_{\text {test }}$ is the PV disturbance along the radial direction. In general, distribution of PV disturbance associated with TC at a specified level does not necessarily have a closed curve of the value with 0 PVU that encloses the TC center (see red contours in Fig. 4 described later); therefore, the condition $q_{\text {test }}<0.05 \mathrm{PVU}$ is sought. Then, the distance of the domain boundary from the TC center is modified to consider the range of $0 \leq q_{\text {test }} \leq 0.05 \mathrm{PVU}$; we add $100 \mathrm{~km}$ to the domain boundary distance assuming that the distance of the range of $0 \leq q_{\text {test }} \leq 0.05 \mathrm{PVU}$ is $100 \mathrm{~km}$. Moreover, the innermost point where the gradient of $q_{\text {test }}$ exceeds $0 \mathrm{PVU} \mathrm{m}^{-1}$ is also regarded as the boundary of TC domain because $q_{\text {test }}$ typically decreases outward. The reason why the starting point for seeking the radial profile is not the TC center but $200 \mathrm{~km}$

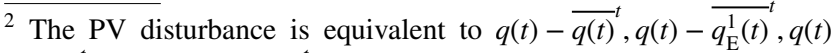
$-{\overline{q_{\mathrm{E}}^{2}(t)}}^{t}, \ldots, q(t)-{\overline{q_{\mathrm{E}}^{n-1}(t)}}^{t}$ in Eq. (9). Note that the PV disturbance varies with the iteration step.
}

$0.2 \mathrm{PVU}$ (red contours indicate $0 \mathrm{PVU}$ ). The blue-colored area represents the TC domain for positive PVA and the three gray-colored circles indicate distances of 500, 1000, and $1500 \mathrm{~km}$ from the TC center

away from the TC center is that the previously defined TC center is not always located at the position of the maximum $q_{\text {test }}$, and thus, the gradient of $q_{\text {test }}$ becomes positive within a range of $0-200 \mathrm{~km}$ in some cases. The distance is bounded by $1200 \mathrm{~km}$, thus the range of the distance is restricted to 200-1200 km. In most cases, the domain boundary exhibits a smoothed curve, but a spike-like curve occasionally appears. The spike-like curve is replaced with the smoothed curve, which is calculated using the adjacent boundary points. The examples of the obtained TC domains are illustrated in Fig. 4. Although the TC domain for a strong storm is almost circular (Fig. 4c), the TC domains for weaker storms can be stretched in certain direction (Fig. 4a, b). Visually, the TC domains can be reasonably detected by this method according to the 0 PVU line shown in Fig. 4.

In some cases, TC couples with other meteorological systems originated in the stratosphere. To prevent picking up the coupled PVAs, we restrict the PVA associated with TC in the troposphere (i.e., the PVA in the stratosphere is not defined as the anomaly associated with TC). In this study, the dynamical tropopause is defined as the surface of $2 \mathrm{PVU}$; the height of the dynamical tropopause is searched downward from the top of the gridded PV data.

As stated above, we considered both positive and negative PVAs as the PVA associated with TC. We assumed $800-\mathrm{km}$ disk from the TC center reported in the best-track data from the surface to the top as the negative PVA domain in this study. This definition is less sophisticated compared with the one of the positive PVA. This is because of highly asymmetric distribution of the negative PVA associated with TCs in most cases. The respective effect of positive and negative 
PVAs associated with TCs on the surrounding large-scale circulations will be discussed later.

\subsection{Estimations of vertical velocity and irrotational wind}

The TC-associated anomalies of nondivergent wind, geopotential, and temperature are obtained from PPVI, and by subtracting these from the respective total fields the TCremoved components are obtained. The TC-removed components of vertical velocity and irrotational wind are calculated based on the quasi-balanced omega equation and continuity equation as in Wang and Zhang (2003) (hereinafter called the balanced omega equation system). In this procedure, diabatic heating and specific humidity, which are given explicitly during the calculation, are modified in the same manner as KF. The Lagrangian derivative of potential temperature is used for the diabatic heating calculation, which is computed from the JRA-55 using centered 6-h air parcel trajectories, that is, 3-h forward and backward trajectories. Five-minute intervals of linear temporal interpolation are considered for the trajectory calculation. Similarly, the TC-included components of the vertical velocity and the irrotational wind are obtained from the balanced omega equation system, but in this instance, the diabatic heating and specific humidity obtained from the JRA-55 is directly used without any modifications. Finally, after subtracting the TC-removed components from the TC-included components, we get the TC-associated vertical velocity and irrotational wind.

\section{Comparison among the effectiveness of PVI and the existing TC removal schemes}

The fields in which TCs were removed by KF at 0000 UTC on August 23, 2004 are shown in Fig. 5. There exist two typhoons: TYs Chaba centered at $15.2^{\circ} \mathrm{N}, 143.6^{\circ} \mathrm{E}$ and Aere centered at $22.9^{\circ} \mathrm{N}, 126.2^{\circ} \mathrm{E}$. The TCs are removed within a polygonal domain at the lower layer (Fig. 5i), but weak cyclonic circulations still exist in the TC-removed field at the location where the TCs originally existed (Fig. 5g, h). Similarly, at the middle layer, the weak cyclonic circulations in the TC-removed field remain (Fig. 5d, e). The isolated TY Aere is not encircled by the zero line of geopotential height deviation (Fig. 5f), indicating that the size of the TC domain at this layer is not sufficient to remove the TC component. TY Aere is elongated southwestwardly (Fig. 5d); therefore, a wider domain is required for the removal. The $\mathrm{TC}$ domain determined by KF is too small because the center and domain are determined at only $850 \mathrm{hPa}$. In addition, the temperature fields at the upper layer are shown in Fig. 5a-c. The warm cores induced by two typhoons seem to be fairly removed in the field. However, similar to the mass and wind fields at the lower and middle layers, high temperature still remains beyond the TC domain in the TC-removed field (Fig. 5a, b).

The TC-removed fields calculated by PVI are shown in Fig. 6. Compared with the results by KF, the TC components of wind and mass fields spread over a wider area and have larger amplitudes (Figs. 5f, i, 6f, i). The TC-removed fields are evidently different from that by $\mathrm{KF}$ and no residual cyclonic circulations at the TC-existed location is found in the TC-removed field (Figs. 5e, h, 6e, h). Whereas the geopotential and wind fields outside the TC domain remain largely unchanged in the results by $\mathrm{KF}$, the outside field exhibits marked changes after the TC removal by PVI. While the TC-removed fields obtained by PVI are dynamically balanced in the PVI framework, it is unclear whether the TC-removed fields obtained by KF are balanced. Similarly, the warm cores associated with the two typhoons obtained from PVI are larger than those obtained from KF (Figs. 5c, $6 \mathrm{c})$; therefore, the high temperature beyond the TC-existed domain shown in the result by KF no longer remains in the PVI result (Figs. 5b, 6b). Note that the maximum temperature at $12^{\circ} \mathrm{N}, 148^{\circ} \mathrm{E}$ in the TC-removed field (Fig. 6b) is associated with another meteorological system, a PV maximum to the southeast of TY Chaba (not shown).

Figure 7 shows relative vorticity of the original and TCremoved fields at the low level. Because of the detection of the larger TC-component wind field by PVI, the relative vorticity of the TC component becomes (approximately $50 \%$ ) larger than that obtained by KF (Fig. 7e, g). Residual amplitudes of relative vorticity still remain at the TC-existed location in the KF result (Fig. 7b). This is because, as shown in Fig. 4 of Kurihara et al. (1995), a weaker cyclonic circulation tends to remain around the TC location when KF is applied. By contrast, such weak anomalies are not found in the TC-removed field by PVI (Fig. 7d). Moreover, in the TC-component vorticity by KF, thick bands of negative vorticity surrounding the positive vorticities centered on the TC centers appear, but such kind of the vorticity structure is not seen in the PVI result (Fig. 7e, g). This tendency of the KF TC detection method in producing thick negative vorticity bands in the surroundings of a TC do not seem realistic. In addition, similar to the TC-removed wind field surrounding the TCs by KF (Fig. 5g, h), the TC-removed vorticity field surrounding TCs remains largely unchanged (Fig. 7a, b). While the environmental wind field surrounding TCs changes more significantly after the TC removal by PVI, the surrounding vorticity field is nearly unchanged as in KF approach, indicating that the choice of TC removal technique (KF or PVI) have a minimum influence on the vorticity in the exterior of the TC region. On the other hand, the result obtained from GDF shows a larger TC effect on the relative vorticity field compared with that by PVI (Fig. 7c, f). This 


\section{Kurihara filter, 00Z23AUG2004}

(a) Total (Temp., 259.3hPa)

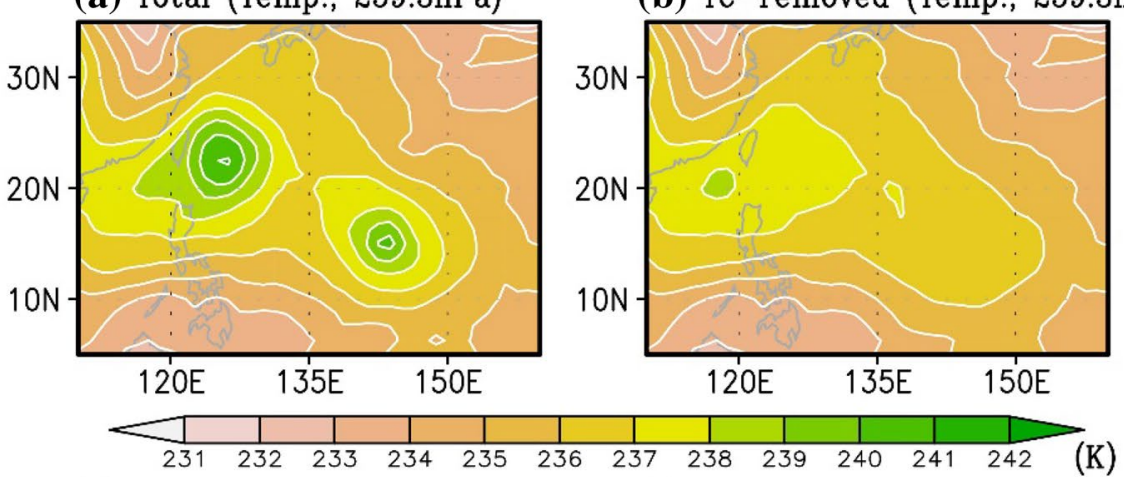

(d) Total (u,v,Z, 499.3hPa)

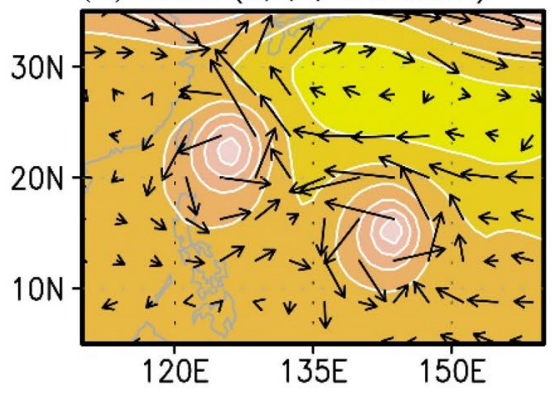

(e) $\mathrm{TC}-$-removed (u,v,Z, $499.3 \mathrm{hPa})$

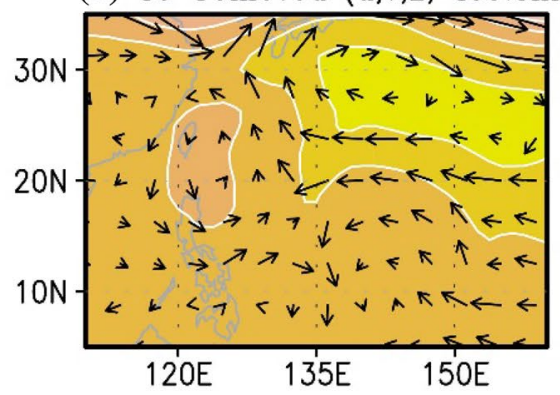

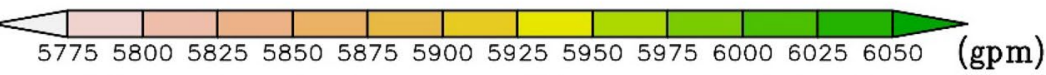

(g) Total $(\mathrm{u}, \mathrm{v}, \mathrm{Z}, 866.9 \mathrm{hPa})$

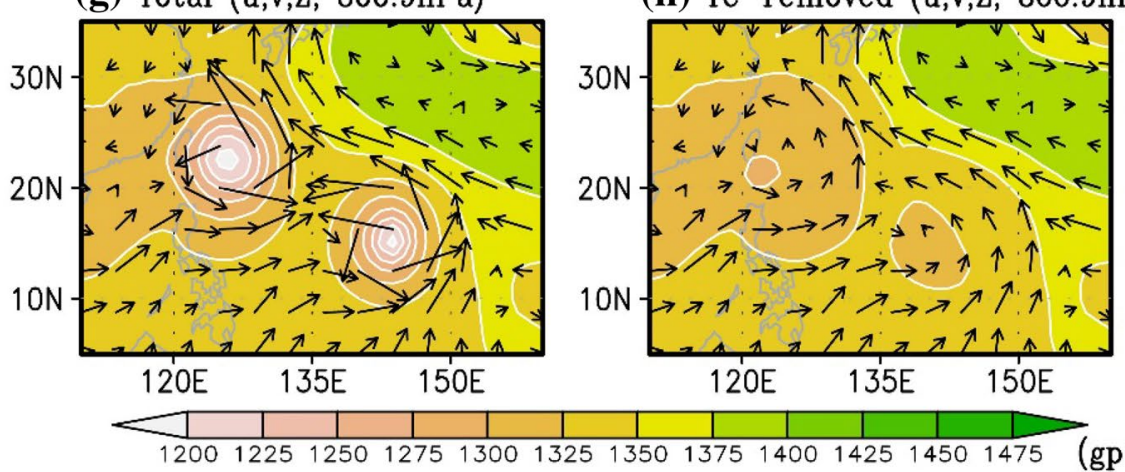

a) (c) TC (Temp., $259.3 \mathrm{hPa})$

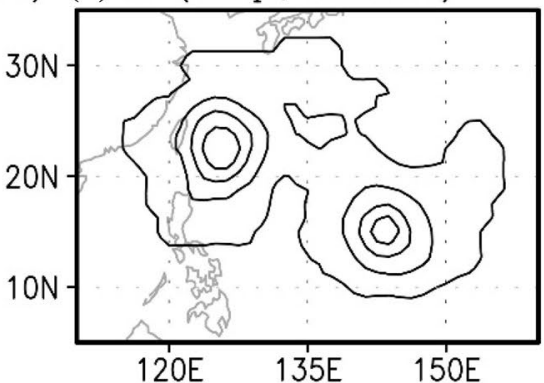
(1)

a) (f) $\mathrm{TC}(\mathrm{u}, \mathrm{v}, \mathrm{Z}, 499.3 \mathrm{hPa})$

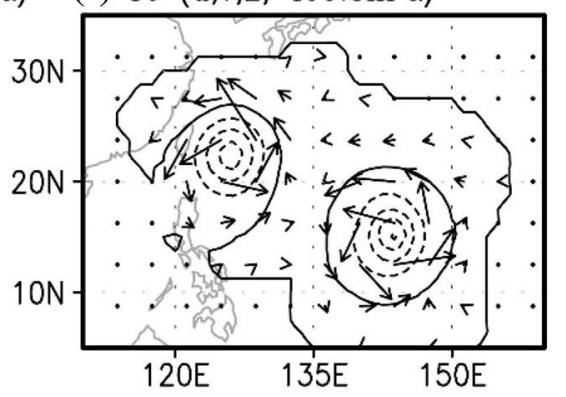

m)

(i) $\mathrm{TC}(\mathrm{u}, \mathrm{v}, \mathrm{Z}, 866.9 \mathrm{hPa})$

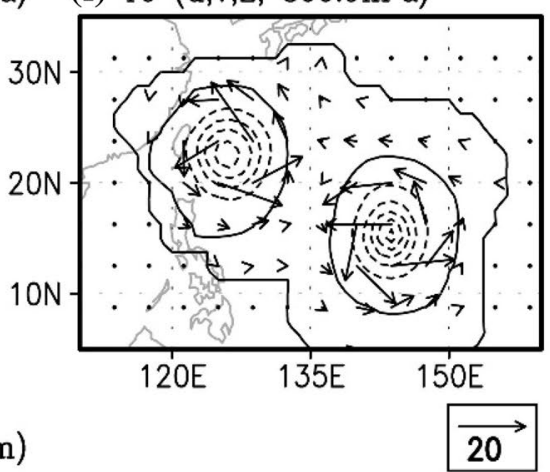

Fig. 5 (Top) Temperature distribution at $259.3 \mathrm{hPa}$ for $0000 \mathrm{UTC}$ on August 23, 2004: a JRA-55 reanalysis; b field in which TCcomponent is removed; and c TC-component. The TC-component is determined based on KF method. (Middle) Same as the top panel but for geopotential height (color and contour) and horizontal wind

is caused by the TC removal procedure in GDF: the vorticity within the defined TC domain is completely removed regardless of the signs (Fig. 7c), thus creating a discontinuity at the boundaries.

To confirm the dynamic consistency of the KF's TCremoved fields, we perform further PVI calculations to their PVAs; the results are shown in Figs. 8, 9, 10. In this calculation, the TC-associated PVA is defined as the difference between the total and TC-removed PV fields obtained by KF (vector) fields at $499.3 \mathrm{hPa}$. (Bottom) Same as the middle panel but at $866.9 \mathrm{hPa}$. Contour interval in the top panel (middle and bottom panels) is $1 \mathrm{~K}(25 \mathrm{gpm})$. A vector scale in units of $\mathrm{m} \mathrm{s}^{-1}$ is shown in the lower right-hand corner

(hereinafter called KF-PVI). The derived TC component is smaller and weaker than the TC component derived from PVI alone (Figs. 6c, f, i, 8c, f, i), but the TC components obtained by KF-PVI alone and those obtained by KF are similar (Figs. 5c, f, i, 8c, f, i). Moreover, as also clearly seen in a vertical cross section, even though the TC components of mass and wind fields obtained by KF and KF-PVI are distributed locally (Fig. 9d, f-i), the components obtained by PVI are distributed over a wider range (Fig. 9e, h). The 


\section{PV inversion, 00Z23AUG2004}

\section{(a) Total (Temp., 259.3hPa)}

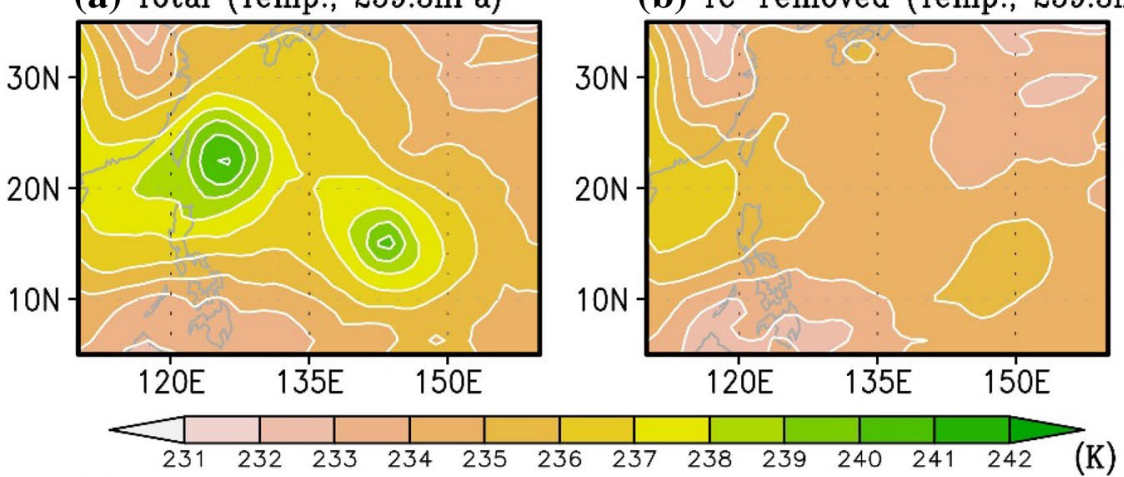

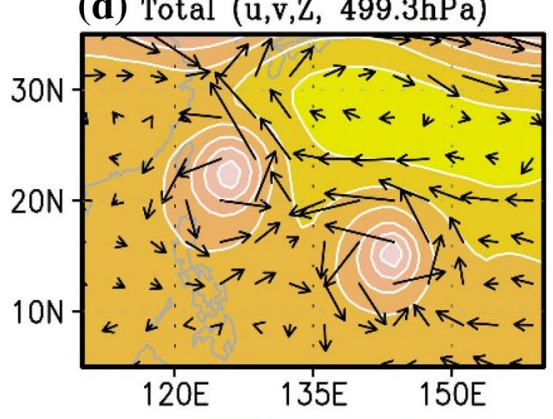

(e) TC-removed (u,v,Z, 499.3hPa)
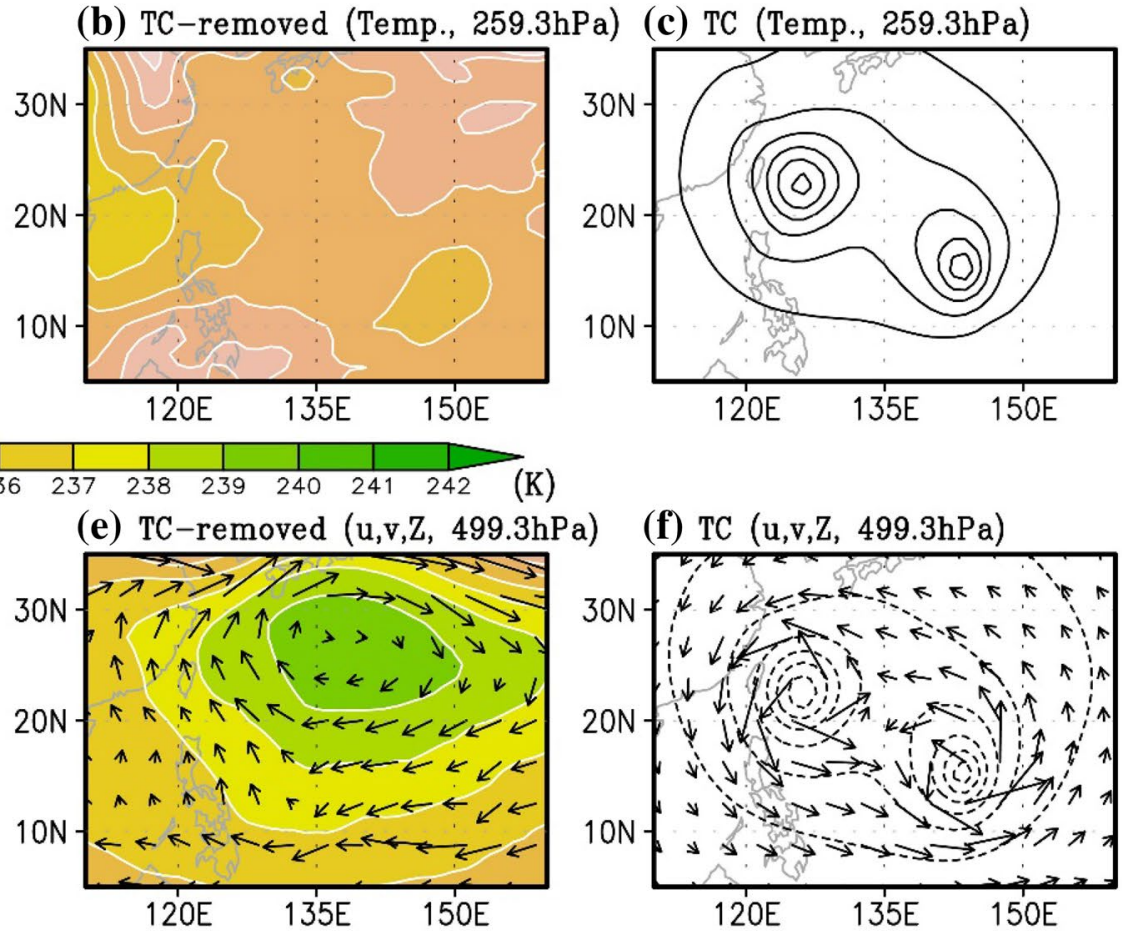

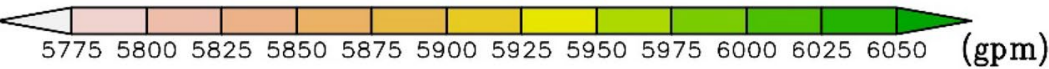

(g) Total $(u, v, Z, 866.9 \mathrm{hPa})$

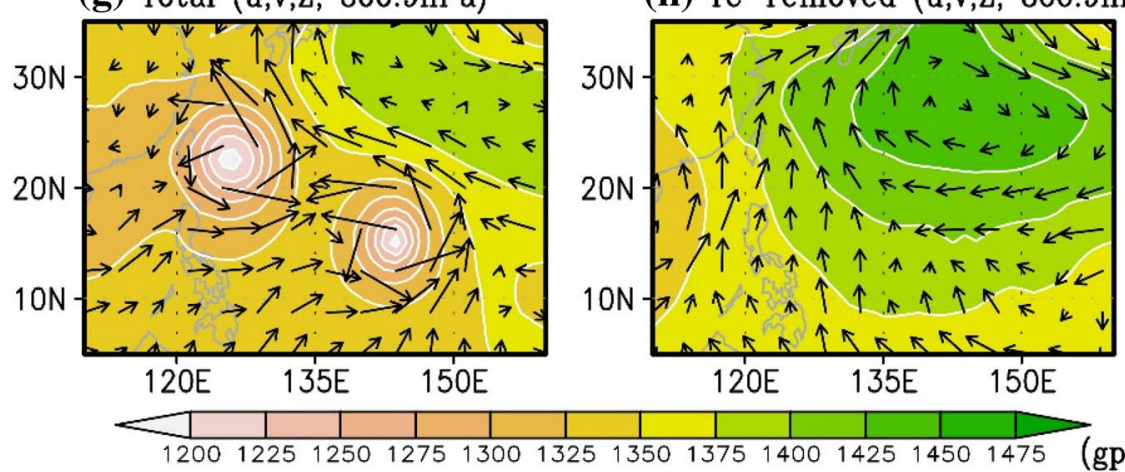

a) (i) $\mathrm{TC}(\mathrm{u}, \mathrm{v}, \mathrm{Z}, 866.9 \mathrm{hPa})$

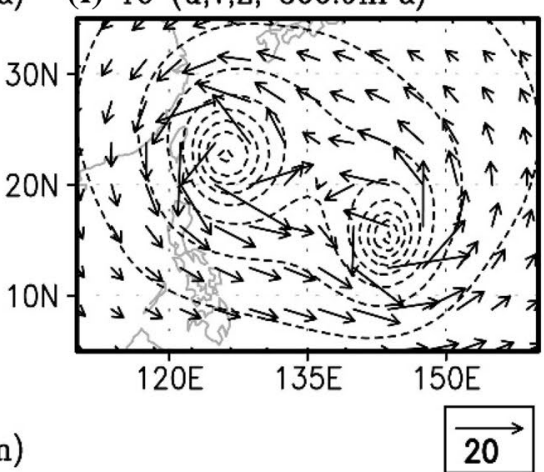

Fig. 6 Same as Fig. 5 but for the results obtained by PVI

TC-removed fields and TC component derived from the KFPVI approach are consistent with the results derived from the KF approach alone, however, the distributions are different from those obtained by PVI.

In principle, PVI would generate a wider-spread geopotential and temperature fields. However, the KF-PVI generates more localized fields than the PVI does (Fig. 9). This seemingly inconsistency is attributed to the cancellation of fields inverted from positive and negative PVAs. To investigate the cancellation, we shows respective effects of positive and negative PVA in KF-PVI in Fig. 10. As same as the vorticity structure shown in Fig. 7e, the TC-associated
PVA in KF has a thick negative PVA surrounding the positive PVA centered on the TC (Fig. 10a). It is obvious that the thick negative PVA is mainly generated by the thick negative vorticity, which is artificially generated by the KF scheme instead of realistic TC characteristic. The positive PVA induces widely-spread TC components of mass and wind fields, which is similar to the PVI result (Fig. 10e, h). On the other hand, the negative PVA induces widely-spread anticyclonic circulation (Fig. 10f, i). The anticyclonic circulation cancels out the widely-spread cyclonic circulation induced by the positive PVA. As a result, the TC components of mass and wind fields are distributed locally. 
$866.866 \mathrm{hPa}$

(a) Total

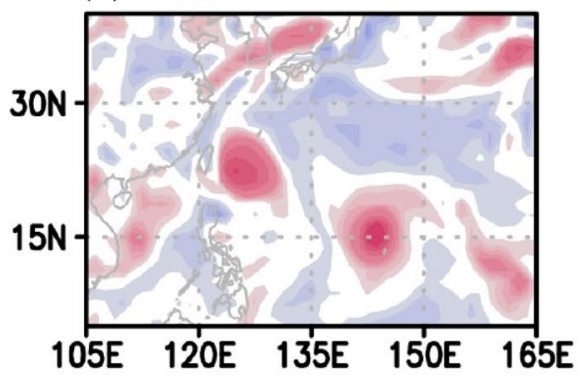

(b) noTC (KF)

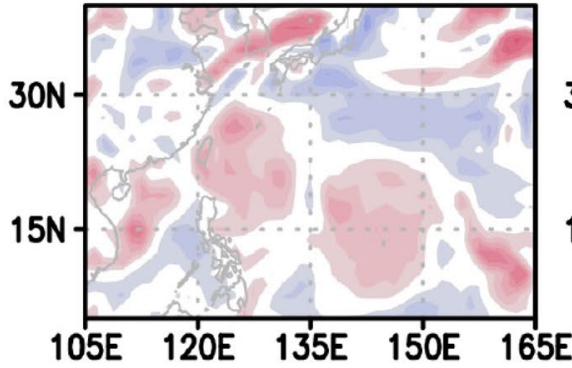

(c) noTC (GDF)

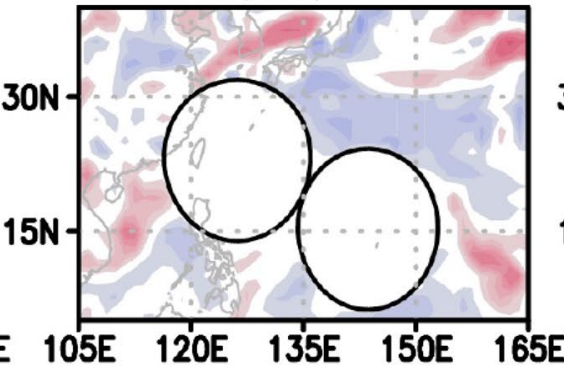

(d) noTC (PVI)

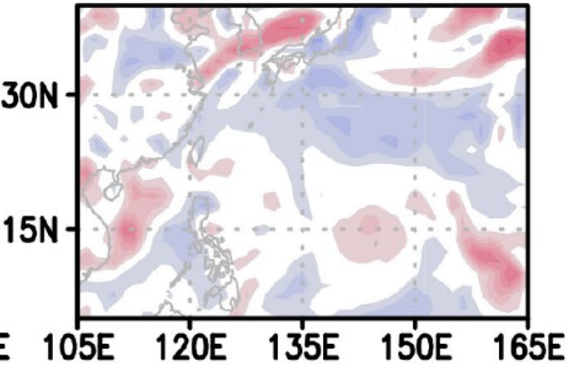

(e) $\mathrm{TC}(\mathrm{KF})$

(f) TC (GDF)
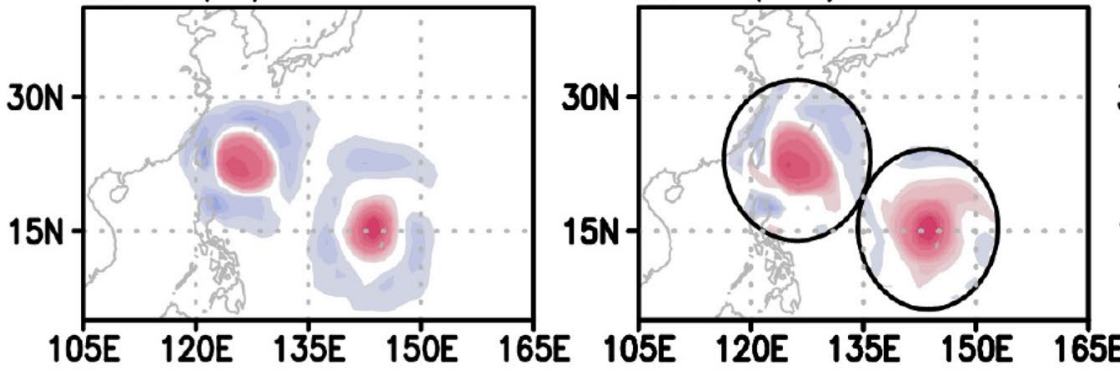

(g) TC (PVI)
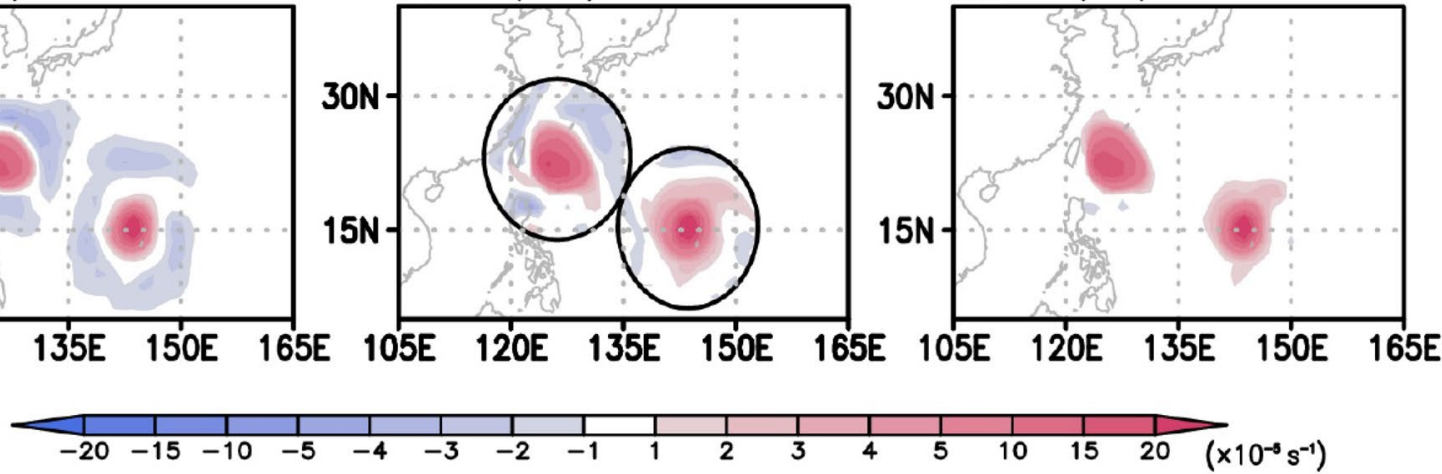

Fig. 7 Relative vorticity (color and contour) at $866.9 \mathrm{hPa}$ for 0000 UTC on August 23, 2004: a JRA-55 reanalysis; b-d field in which TC-component is removed; and $\mathbf{e}-\mathbf{g}$ TC-component. The fields are

The PVI approach can be used to derive the mass and temperature associated with the TC-removed vorticity obtained from GDF. Figure 11 shows the result of PVI calculation, in which the PVAs are calculated using the TC-removed fields obtained by GDF [here, to compute PV in the TC-removed field, temperature in the TC-removed field is obtained by the spatial filtering used in Kurihara et al. (1995)]. The derived TC components are slightly smaller but qualitatively similar to the result derived by PVI (Figs. 9e, h, 11b, c). This indicates that GDF fairly calculates TC-associated PVA and confirms again that the mass and temperature fields associated with TC vorticity exhibit a wider spread structure than the vortex itself. However, a direct application of this removal technique decomposed by b, e KF, $\mathbf{c}, \mathbf{f}$ GDF, and $\mathbf{d}, \mathbf{g}$ PVI. The circles shown in c, $\mathbf{f}$ indicate the boundaries of TC removal regions

needs to be cautioned because the TC component of relative vorticity decomposed by GDF includes background relative vorticity (Fig. 7c).

\section{Contribution of TCs to intraseasonal variability and seasonal-mean fields in the typhoon season in 2004}

The technique proposed here is potentially useful for quantifying the contribution of TCs to climate variability. Two examples, namely seasonal-mean field and intraseasonal oscillation in JJASO 2004, are demonstrated here. Figure 12 shows the seasonal-mean relative vorticity of the original 


\section{$\mathrm{KF} \rightarrow \mathrm{PVI}, \quad 00 \mathrm{Z} 23 \mathrm{AUG} 2004$}

\section{(a) Total (Temp., 259.3hPa)}

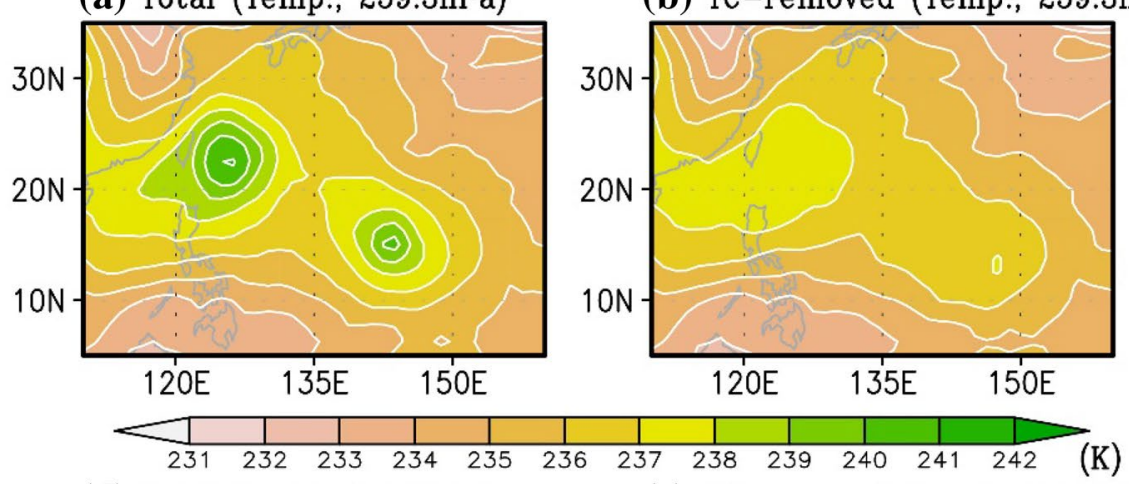

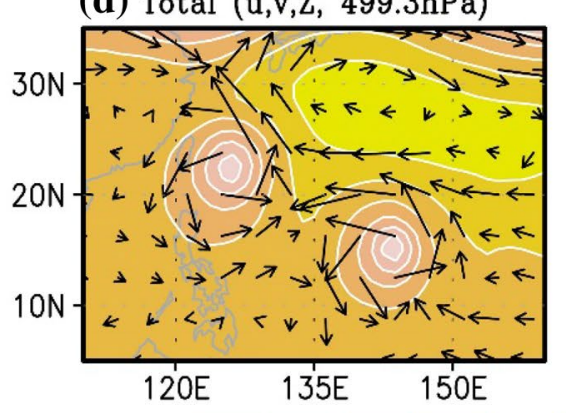

(e) $\mathrm{TC}-$ removed $(\mathrm{u}, \mathrm{v}, \mathrm{Z}, 499.3 \mathrm{hPa})$
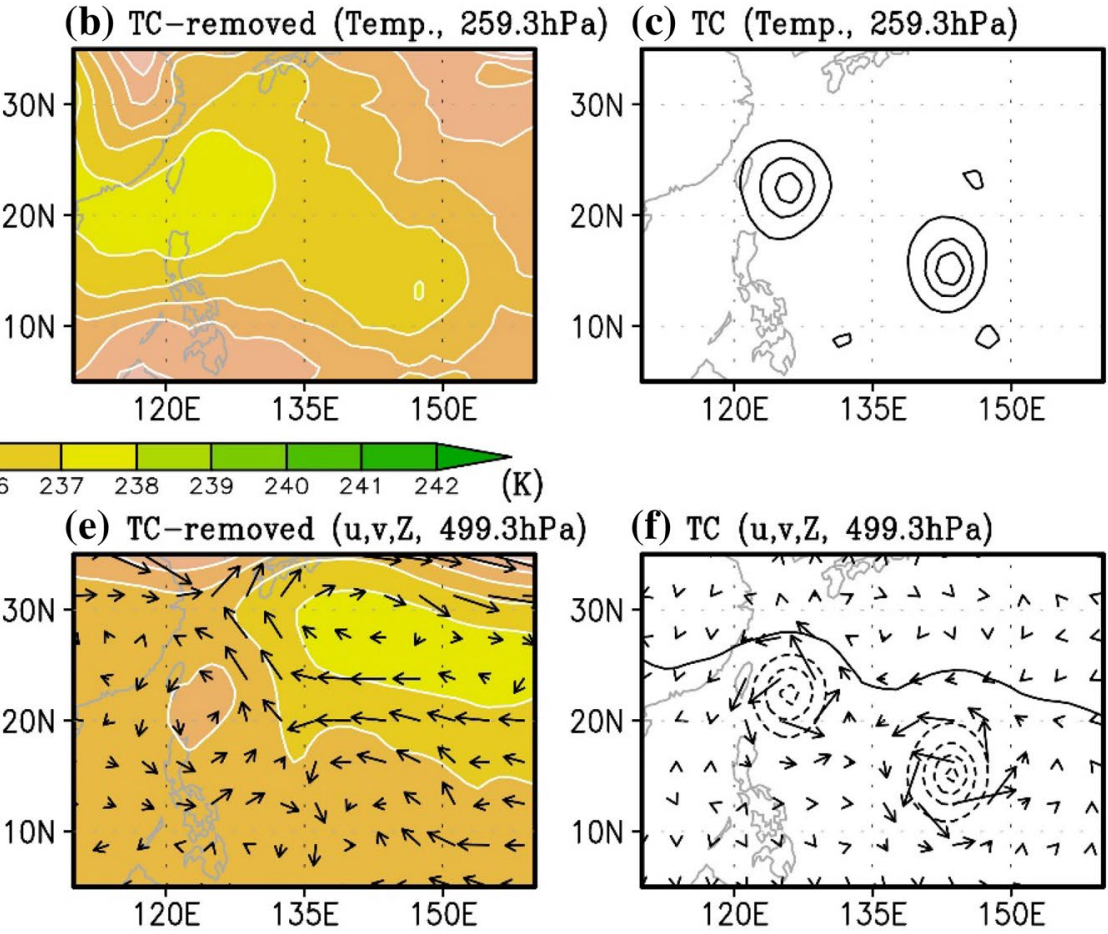

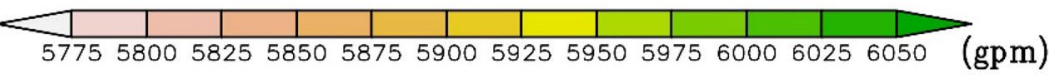

(g) Total $(\mathrm{u}, \mathrm{v}, \mathrm{Z}, 866.9 \mathrm{hPa})$

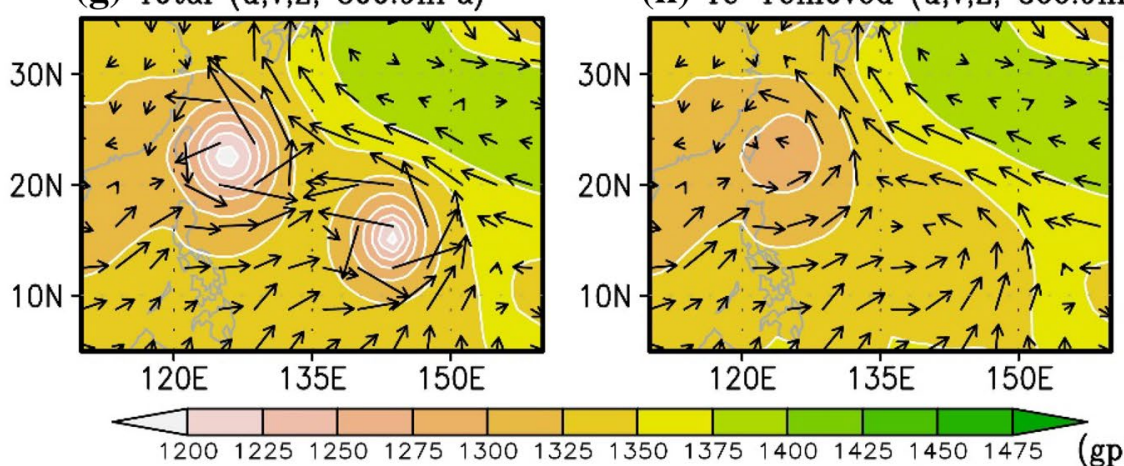

$\mathrm{Pa}$

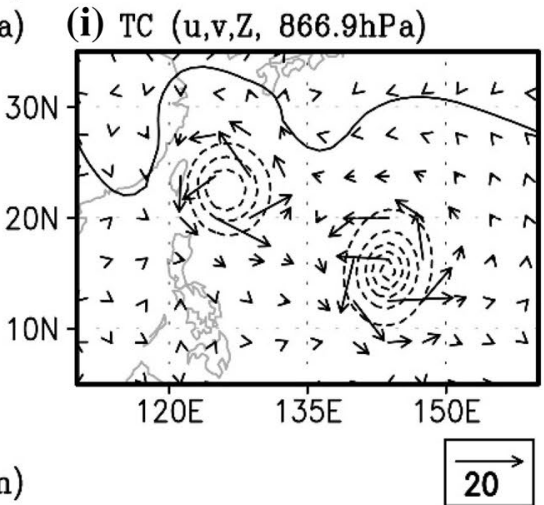

Fig. 8 (Left-hand and center columns) Same as the center and the right-hand columns of Fig. 5 but the input PVA is calculated using the TC-removed fields obtained by KF, respectively. (Right-hand column) Difference of the TC component (center column) and the TC component obtained by KF (the right-hand column of Fig. 5). Contour intervals in panel $\mathbf{c}$ and $\mathbf{f}, \mathbf{i}$ are $0.25 \mathrm{~K}$ and $25 \mathrm{gpm}$, respectively. A vector scale in units of $\mathrm{m} \mathrm{s}^{-1}$ is shown in the lower right-hand corner

north and smaller positive vorticity in the south. Moreover, consistent with the 6-h vorticity fields shown in Fig. 7, the TC component of seasonal-mean vorticity obtained by PVI is approximately $40 \%$ larger than that derived from KF (Fig. 12e, g). Because of the larger vorticity of the TC component derived from PVI, the positive vorticity in the central Philippine Sea becomes smaller, and the negative vorticity in the northeastern of the recurving point becomes larger than by KF (Fig. 12b, d). On the other hand, the TC component of Removal of TC PV results in larger negative vorticity in the 
(a) $\mathrm{KF}\left(\mathrm{q}^{\prime}\right)$

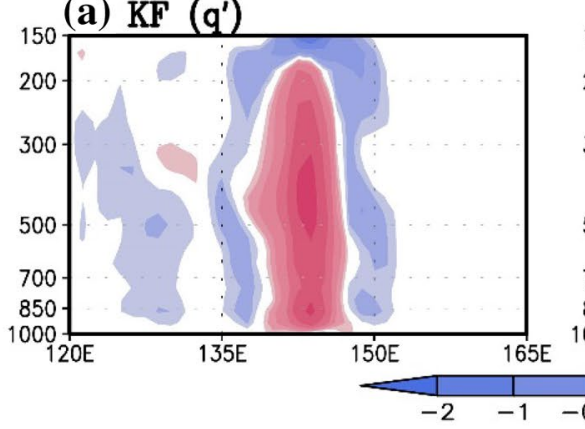

(b) PVI $\left(q^{\prime}\right)$

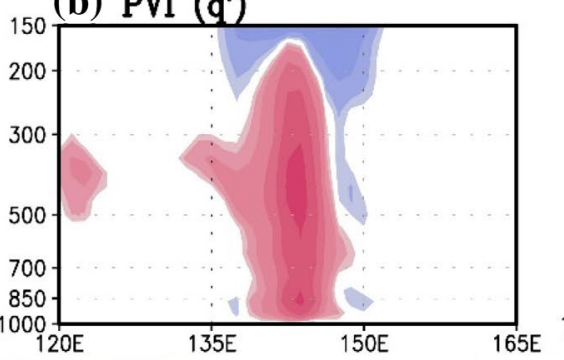

(c) $\mathrm{KF} \rightarrow \mathrm{PVI}\left(\mathrm{g}^{\prime}\right)$

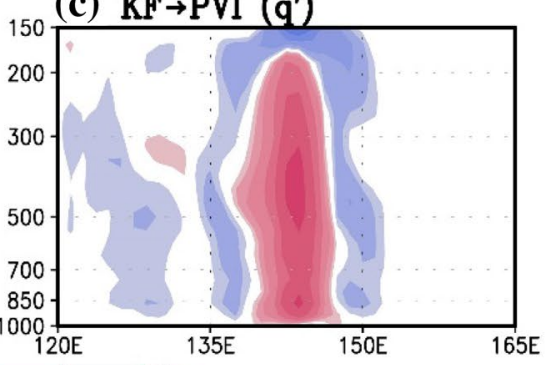

(d) $\mathrm{KF}\left(\mathrm{z}^{\prime}\right)$

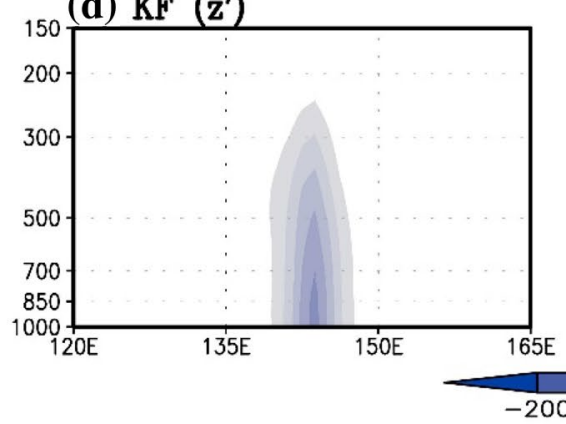

(e) PVI ( $\left.\mathrm{z}^{\prime}\right)$

(f) $\mathrm{KF} \rightarrow \mathrm{PVI}\left(\mathrm{z}^{\prime}\right)$

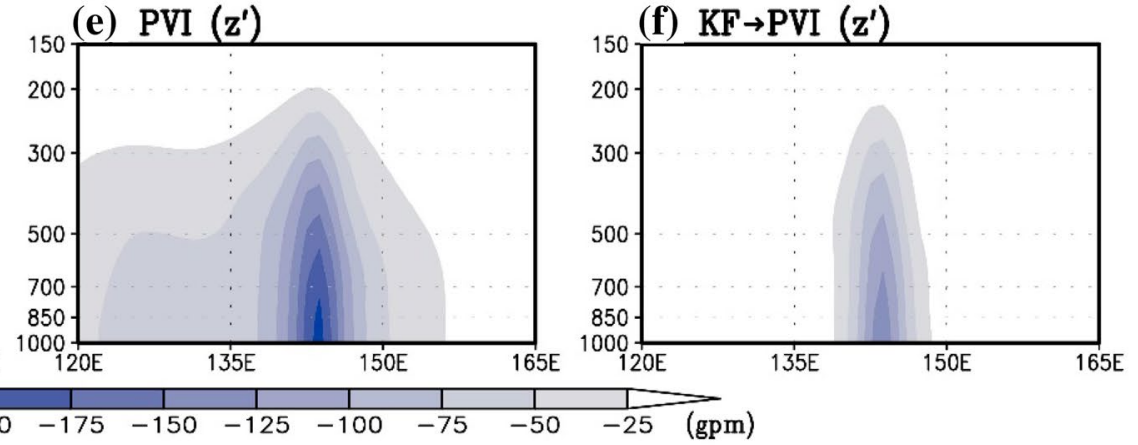

(g) KF ( $\left.\mathbf{v}^{\prime}\right)$

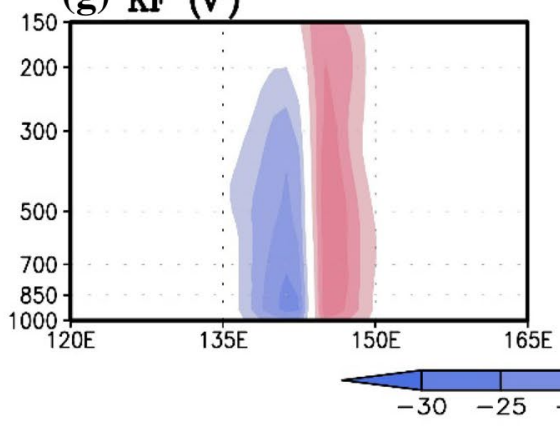

(h) PVI $\left(\mathbf{v}^{\prime}\right)$

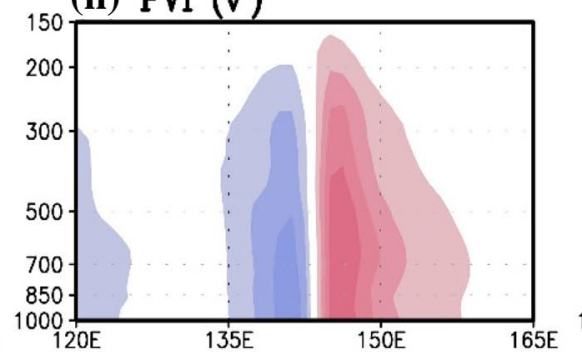

(i) $\mathrm{KF} \rightarrow \mathrm{PVI}\left(\mathrm{v}^{\prime}\right)$

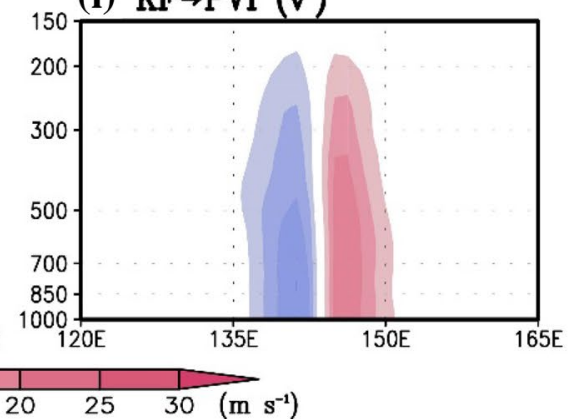

Fig. 9 Vertical cross section at $15^{\circ} \mathrm{N}$ for $0000 \mathrm{UTC}$ on August 23, 2004 of TC-associated (top) PVA, (middle) geopotential height deviation, and (bottom) meridional wind deviation. The left-hand (center) column is derived from the result obtained by KF (PVI) [correspond-

seasonal-mean vorticity obtained by GDF is approximately $20 \%$ larger near the statistical recurvature point than that derived from PVI (Fig. 12f, g). This results in the larger negative vorticity in the northeastern of the recurving point than those based on PVI (Fig. 12c, d). GDF's larger TC contribution to the seasonal-mean vorticity is likely caused by the removal of not only the TC component of the relative vorticity within the removal domain but also its background field in the scheme.

Figure $13 \mathrm{a}-\mathrm{c}$ shows the seasonal-mean upper-level temperature of the original and TC-removed fields. After removal of TCs, the seasonal-mean upper-level temperature becomes lower in both KF and PVI results. The largest temperature decrease exists to the east of Taiwan (approximately ing to Fig. 5c, f, i (Fig. 6c, f, i)]. The right-hand column is the result by PVI but the input PVAs are calculated using KF's TC-removed fields (corresponding to Fig. 8b, e, h)

$25^{\circ} \mathrm{N}, 128^{\circ} \mathrm{E}$ ). Because larger warm core is calculated in PVI than in KF, a temperature decrease in the upper-level temperature after removal of TCs by PVI is larger than that by KF; the temperature decrease in KF and PVI is 0.35 and $1.35 \mathrm{~K}$, respectively, at the largest temperature decrease location. The seasonal-mean low-level geopotential height of the original and TC-removed fields are shown in Fig. 13d-f. While a weak seasonal-mean cyclone remains to the east of Philippines becomes smaller after TC removal by KF, the seasonal-mean cyclone totally disappears in PVI result. The extent of low-level negative vorticity in the northeastern of the recurvature point obtained from GDF and PVI (Fig. 12c, d) indicates that the subtropical anticyclone expands its spatial coverage westward and becomes more dominant in 


\section{Vertical cross section at $15.00^{\circ} \mathrm{N}, 00 \mathrm{Z} 23 \mathrm{AUG} 2004$}
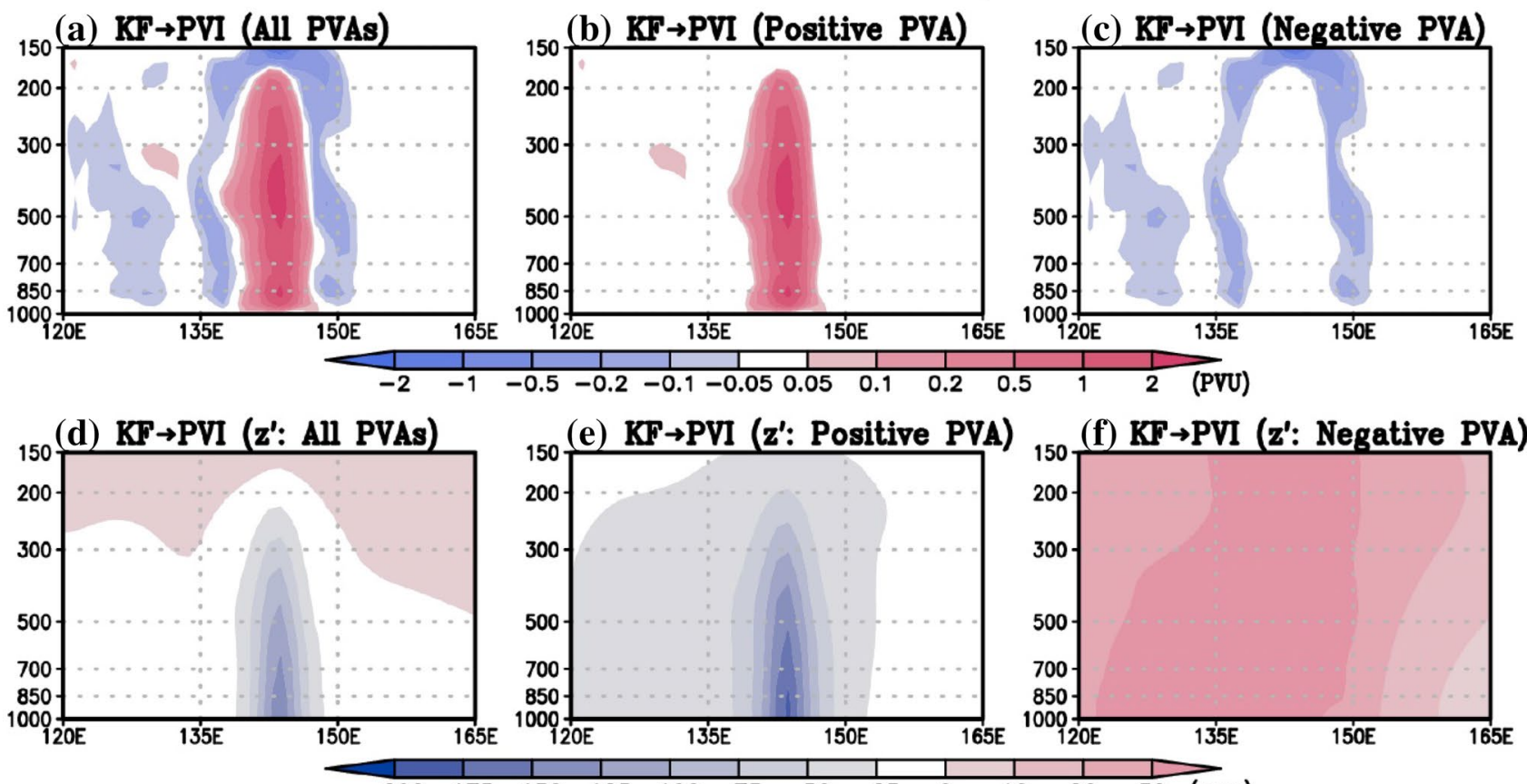

(e) KF $\rightarrow$ PVI (z': Positive PVA)

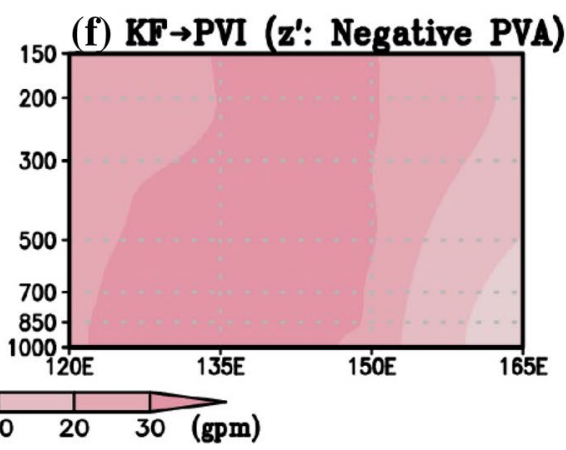

(g) KF $\rightarrow$ PVI ( $\mathbf{v}$ : All PVAs)
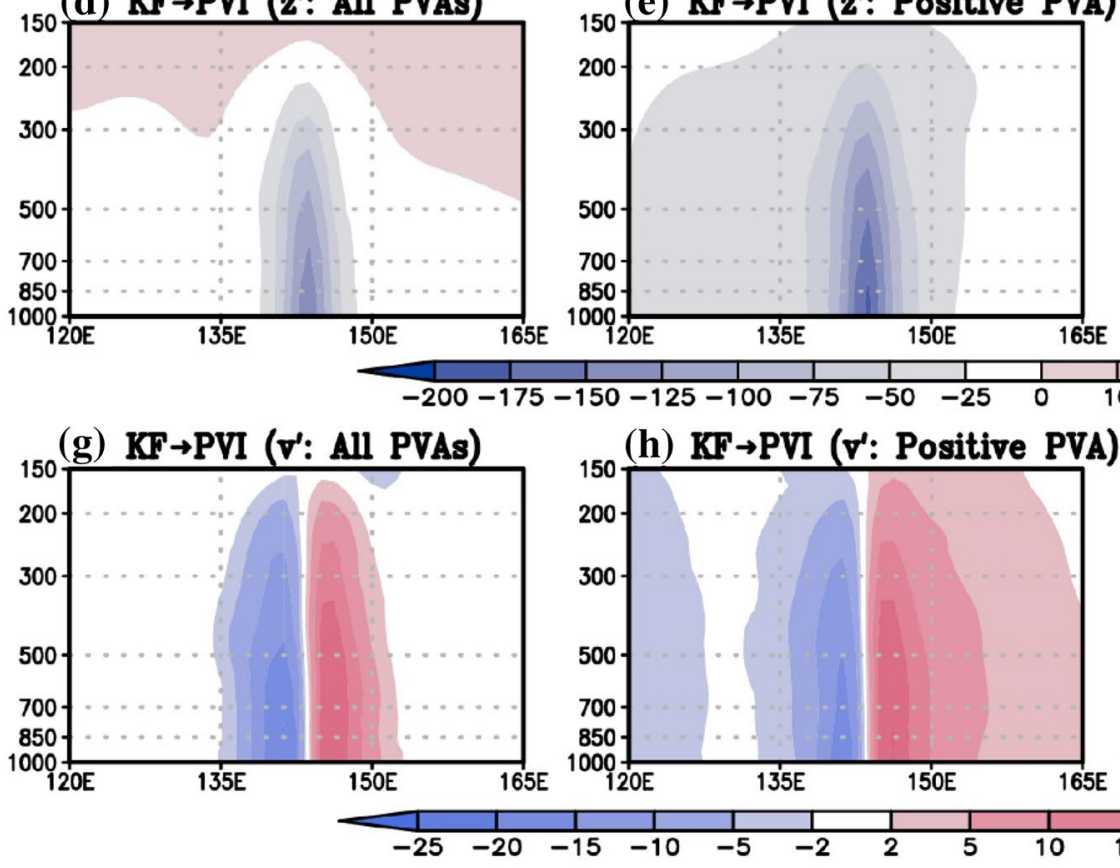

(h) KF $\rightarrow$ PVI ( $\nabla^{\prime}:$ Positive PVA)

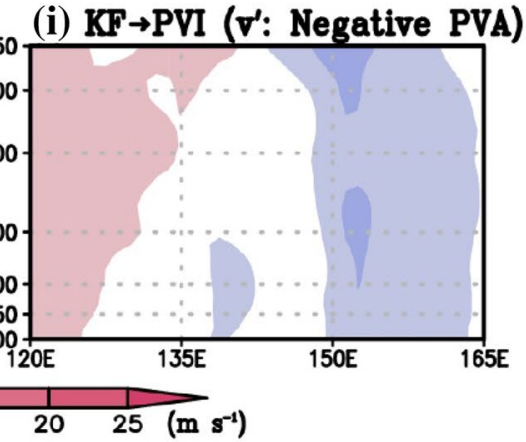

Fig. 10 (Left-hand column) Same as Fig. 9c, f, i with different color ranges. (Center column) Same as the left-hand column but the results are induced by positive PVA. (Right-hand column) Same as the left-hand column but the results are induced by negative PVA

the subtropical WNP after TC removal. Clearly, as would be expected from the 6-h TC-removed geopotential height fields (Fig. 6e, h), the seasonal-mean TC-removed geopotential height field shows the westward expansion and intensification of anticyclone in the region (Fig. 13d, f). The above results reveal that the existence of TCs with their strong positive vorticity enhances the strength of the monsoon trough and weakens the subtropical anticyclone in the lower troposphere, and at the same time warms the troposphere. In other words, the strength of seasonal-mean circulations in the tropical WNP is markedly enhanced because of the presence of TCs, which are induced in the favorable background conditions provided by the large-scale circulations. This result clearly demonstrates the mutual dependence of TCs and background mean circulations through active interaction.
Hsu et al. (2008a) revealed that the 32-76-day ISO was the dominant subseasonal fluctuation in JJASO 2004, and its amplitude reached the 50-year record high value. The contributions of TCs to the 32-76-day ISV at the lower layer were estimated for both TC-removed fields, as shown in Fig. 8 by Hsu et al. (2008a), and the difference between these contributions were also investigated. The contributions of 32-76-day variance of the low-level vorticity indicate two major ISV regions along the TC tracks: one is located to the east of Taiwan and the northern Philippines, and the other to the south of Japan (Fig. 14e). The former corresponds to the northwestward TC tracks, while the latter corresponds to the northeastward TC tracks after recurvature. As compared with Fig. 8 by Hsu et al. (2008a), the variance associated with the East Asian monsoon trough in the South China Sea is much larger than the variance associated with the 


\section{Vertical cross section} at $15.00^{\circ} \mathrm{N}$

(a) GDF $\rightarrow$ PVI $\left(q^{\prime}\right)$

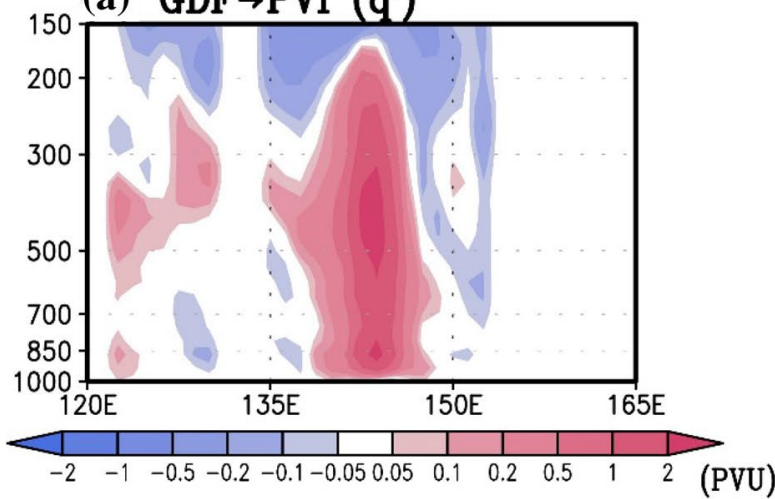

(b) GDF $\rightarrow$ PVI ( $\left.\mathbf{z}^{\prime}\right)$

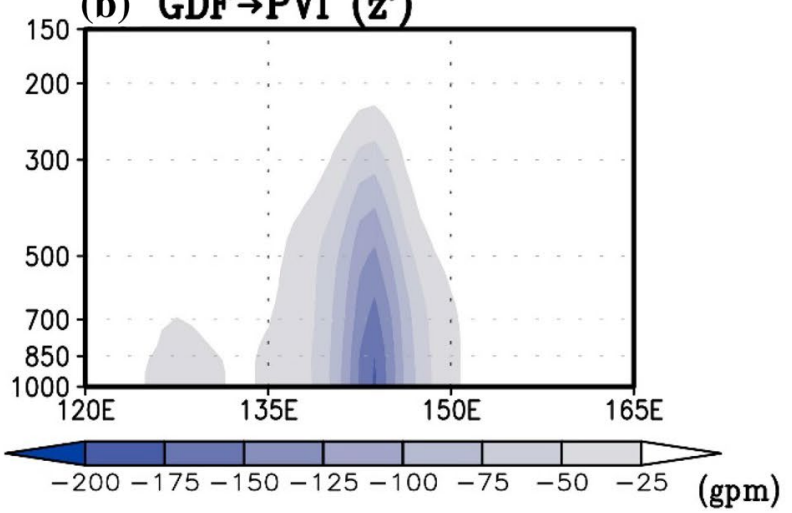

(c) GDF $\rightarrow$ PVI $\left(v^{\prime}\right)$

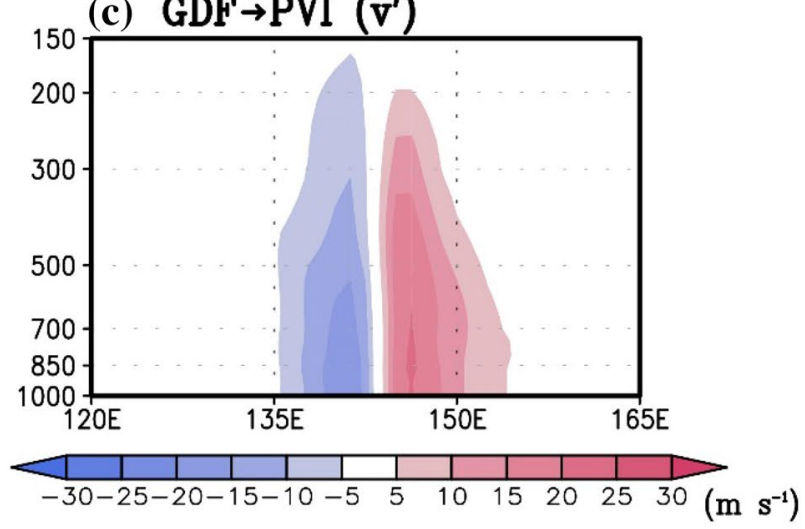

Fig. 11 Same as the right-hand column of Fig. 9 but the input PVAs are calculated using GDF's TC-removed fields

clustered TCs (Fig. 14a). This is because of the differences in the resolution $\left(1.25^{\circ}\right.$ versus $\left.2.5^{\circ}\right)$ and temporal interval ( $6 \mathrm{~h}$ versus daily) of the data used in the analysis, as discussed in Sect. 5 of Hsu et al. (2008b). The TC contribution obtained by GDF is consistent with KF's but in much larger magnitude (Fig. 14f). The stronger TC vorticity derived from GDF presumably leads to the larger TC contribution to the subseasonal variance. The difference between the contributions calculated by KF and PVI appears in the northwestward-track region: the TC contribution calculated by PVI in the northwestward-tracks region is larger (up to $20 \%$ ) than the contribution calculated by KF (Fig. 14g). By contrast, the differences in the TC contribution are relatively small in the northeastward-track region. The variance reduced significantly in these two regions after removing the TCs (Fig. 14a-d). Similar to the result in Hsu et al. (2008a), the clustered TCs contribute $50 \%$ or more to ISV along the TC tracks in both TC removal procedures, and this contribution is larger when PVI is applied.

To investigate the ISO propagation after the removal of TCs, the Hovmöller diagrams of low-level vorticity are shown in Fig. 15. The ISO propagates northward associated with the northward movement of TCs; therefore, we first show the Hovmöller diagrams of low-level vorticity averaged over $120^{\circ}-150^{\circ} \mathrm{E}$ in Fig. 15a. The large amplitudes are observed near $8^{\circ}$ and $17^{\circ} \mathrm{N}$, and northward propagation from $5^{\circ}$ to $20^{\circ} \mathrm{N}$ is also evident. The intraseasonal perturbation repeatedly oscillated throughout JJASO 2004. After the TC removal, the large amplitudes of the vorticity weaken significantly between $10^{\circ}$ and $25^{\circ} \mathrm{N}$ (Fig. 15b-d). Spectral analysis reveals that removing the TCs reduces the intraseasonal amplitude by $50 \%$ (not shown). The northward propagation becomes much weaker and less systematic after TC removal. More interestingly, a slow southward propagation from $30^{\circ}$ to $10^{\circ} \mathrm{N}$, which is not present before TC removal, becomes evident. Thus, the TCs leave marked imprints in the characteristics of the ISO, not only enhancing the amplitude but also over-dominating the propagation tendency of largescale intraseasonal perturbation. These changes in the ISO characteristics can be seen in all three TC removal methods; however, the PVI method yields larger differences.

Next, because ISO is mainly affected by the northwestward TC tracks in the $10^{\circ}-20^{\circ} \mathrm{N}$ latitudinal band, to investigate longitudinal propagation, we show the Hovmöller diagrams of low-level vorticity averaged over $10^{\circ}-20^{\circ}$ $\mathrm{N}$ in Fig. 15e. The eastward propagation of the low-level vorticity from $120^{\circ}$ to $160^{\circ} \mathrm{E}$ is clearly observed from the middle of July 2004. Intraseasonal oscillations are also evident near $120^{\circ}, 130^{\circ}$, and $145^{\circ} \mathrm{E}$. After the TC removal, the amplitudes of the vorticity and oscillations weaken, especially between $120^{\circ}$ and $160^{\circ} \mathrm{E}$, where TCs originally existed (Fig. 15f-h). As with the northward propagation, the eastward propagation becomes less evident after the TC removal. Although the choice of the TC removal method influences the vorticity amplitude, the effect is weaker than that in the northward component. Overall, the presence of TCs not only enhances amplitude but also contributes to the more organized propagation of the ISO. 


\section{$866.866 \mathrm{hPa}$}

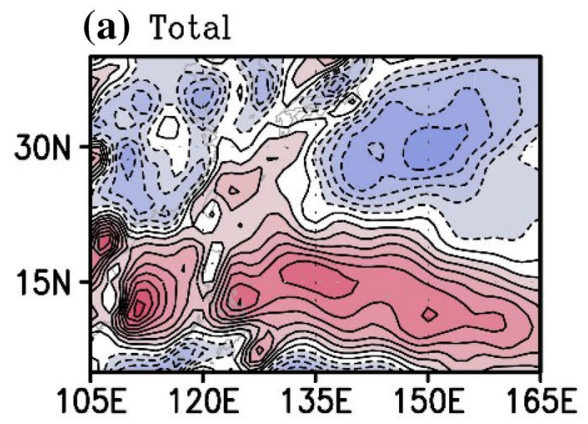

\section{(b) noTC (KF)}

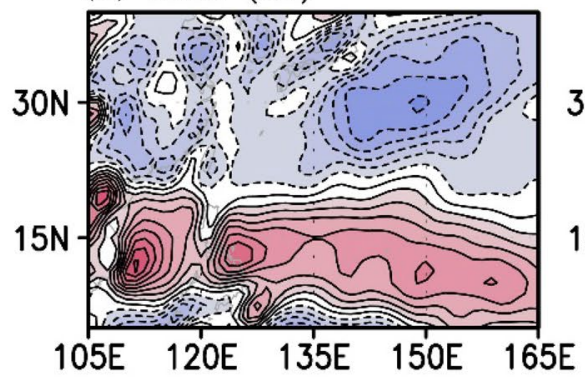

(e) $\mathrm{TC}(\mathrm{KF})$

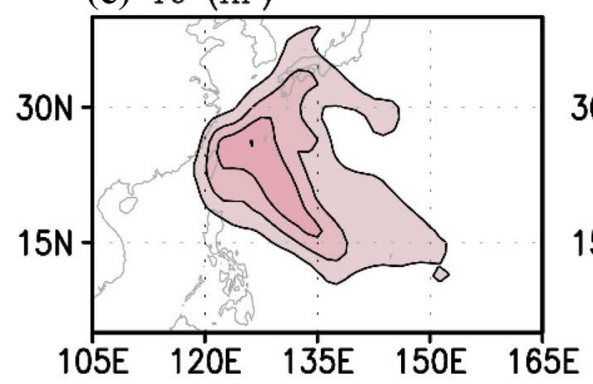

(c) noTC (GDF)

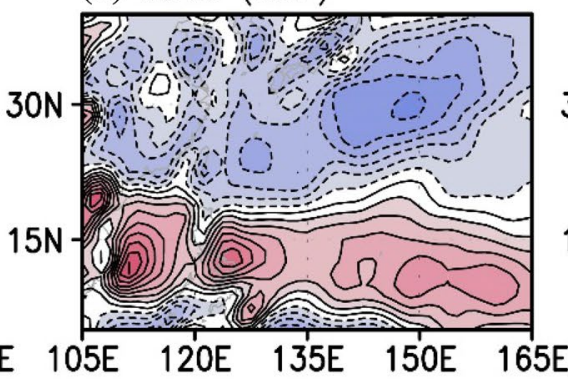

(f) $\mathrm{TC}$ (GDF)

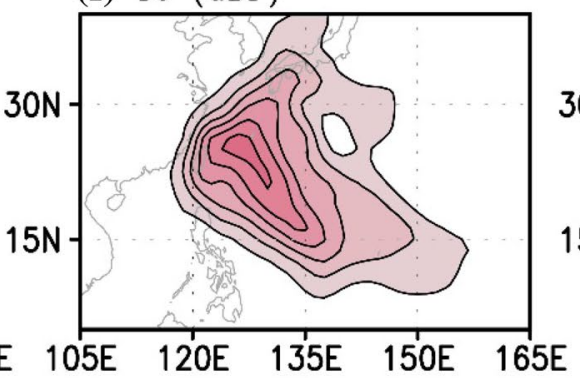

(d) noTC (PVI)

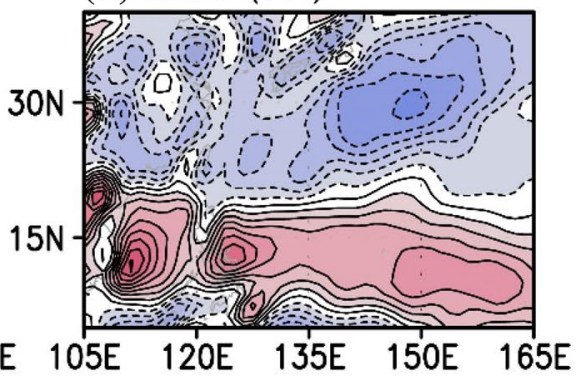

(g) TC (PVI)

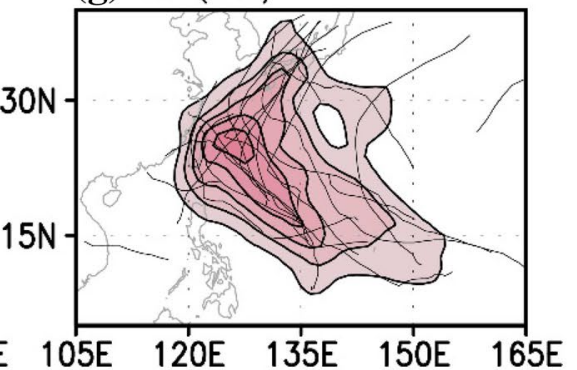

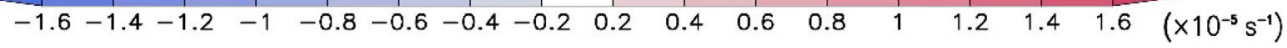

Fig. 12 Same as Fig. 7 but for the time mean in JJASO 2004. Contour interval is $0.2 \times 10^{-5} \mathrm{~s}^{-1}$. The TC tracks during JJASO 2004 are also shown in $\mathbf{g}$

\section{Respective effects of positive and negative PVAs associated with TC}

The effect of negative PVA in TC is evaluated here. The contributions by tropospheric positive PVA and tropospheric and stratospheric negative PVAs for the wind, geopotential, and temperature fields during JJASO 2004 are calculated by PVI. Hereinafter, we refer to the PVI calculation considering only positive PVA as P-PVI and the original calculation considering both positive and negative PVAs as PN-PVI.

Figure 16 shows that TC-removed fields on August 23, 2004 obtained by P-PVI, and the corresponding vertical structures of PN-PVI and P-PVI results are compared in Fig. 17. Although the expansion of subtropical anticyclone is observed as in the TC-removed fields obtained by PN-PVI (Fig. 6), the magnitude of the anticyclone is stronger than the PN-PVI result (Fig. 16e, f, h, i). This is because upper-level negative PVA remotely induces anomalous anticyclonic circulation in the lower troposphere and partially cancel out the anomalous low-level cyclonic circulation induced by positive PVA in PN-PVI (Figs. 6f, i, 16f, i, and 17c, d). Evidently, the effect of upper-level negative PVA, which is weaker and vertically thinner, is relatively weaker than the effect of positive PVA, which is stronger and extends vertically through almost the whole troposphere. As a result, the strengthening and expansion of subtropical anticyclone is still dominant even after removing both positive and negative PVAs associated with TCs. 


\section{3hPa, Temperature}

(a) Total

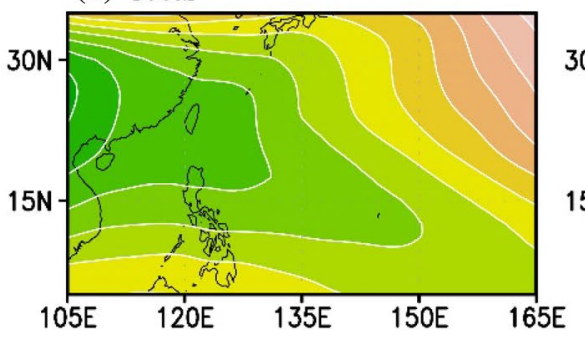

(b) notC (KF)

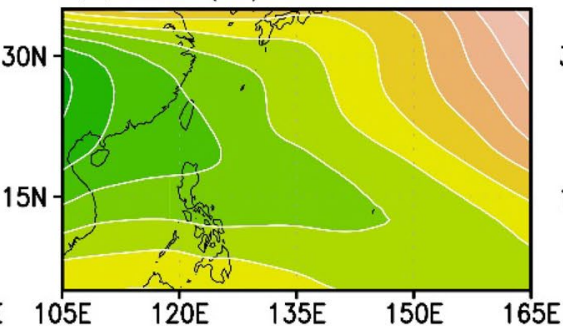

(c) noTC (PVI)

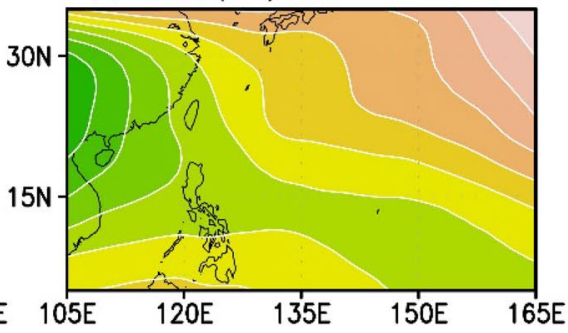

866.9hPa, Geopotential Height

(d) Total

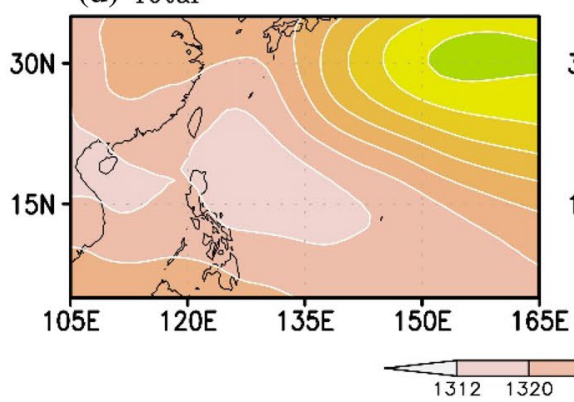

(e) noTC (KF)

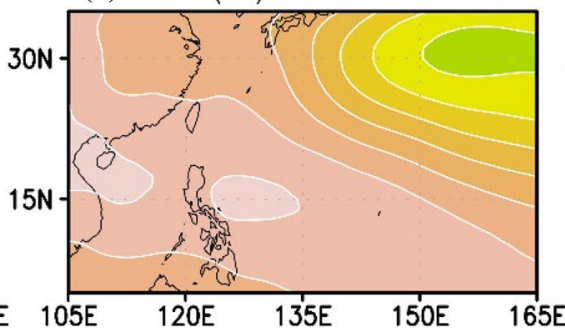

(f) notC (PVI)

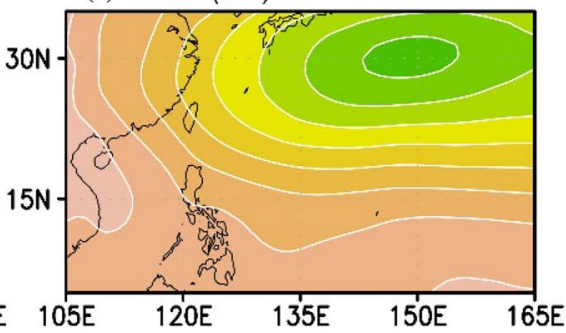

Fig. 13 Temperature (color and contour, in $\mathrm{K}$ ) at $259.3 \mathrm{hPa}$ for the time mean in JJASO 2004: a JRA-55 reanalysis; TC-removed field obtained by $\mathbf{b} \mathrm{KF}$ and $\mathbf{c}$ PVI. d-f Same as a-c but for geopotential height at $866.9 \mathrm{hPa}$ (in gpm)

Next, we examine the upper-level TC-removed temperature field. The TC-component of upper-level temperature is smaller in the P-PVI calculation than in the PN-PVI calculation (Figs. 6c, 16c, and 17e, f), resulting in the relatively higher temperature region in the TC-removed field near the TC center (Figs. 6b, 16b). To investigate the upper-level temperature in detail, the decomposed effect of PVAs are shown in Fig. 18. Figure 18 indicates that the negative PVA in the troposphere induces not only an anticyclonic circulation but also a cold layer in the lower stratosphere and a warm layer in the upper troposphere (Fig. 18e, h). The temperature anomaly induced in the cold and warm layers are $-1.5 \mathrm{~K}$ and $1.5 \mathrm{~K}$, respectively, thus the negative PVA effect on upper-level temperature fields is not small. By contrast, the positive PVA induces cyclonic circulation throughout most of the troposphere with maximum in the low level, and a deep warm core in the troposphere. The effect of negative PVA in the lower stratosphere on the circulation in the middle-lower troposphere is relatively minimum compared with that of positive PVA (Fig. 18c, f, i). The net effect of both positive and negative PVAs associated with a TC appears to enhance the background large-scale circulations, namely a stronger low-level monsoon trough, a stronger upper-level anticyclone, and a warmer core throughout the troposphere.
The effect of the thinner upper-level negative PVA is essentially limited in the upper level, whereas the deep positive PVA has a much larger and deeper influence.

Finally, the seasonal-mean upper-level temperature and low-level geopotential height in the TC-removed fields are shown in Fig. 19. Because of weaker warm core structure obtained by P-PVI (Fig. 17e, f), smaller temperature reduction is observed compared with that caused by $\mathrm{PN}$ PVI (Fig. 19b, c). Moreover, compared with the result in PN-PVI, the seasonal-mean low-level anticyclone becomes stronger in P-PVI because there is no consideration of the negative PVA that suppresses the expansion of subtropical anticyclone (Fig. 19e, f). On the other hand, the TC's low-level contribution to the 32-76-day ISV and the ISO propagation in the TC-removed field obtained by P-PVI are almost the same as the results obtained by PN-PVI because the negative PVA effect on low-level wind field is much weaker (not shown).

In conclusion, removing positive PVA alone might be essentially enough for estimating the TC contribution on low-level circulation. However, the negative PVA should be taken into account because the TC contribution induced by the negative PVA especially on the upper level temperature field is not small. 
(a) Total (Cont. intv. $=2.5 \times 10^{-11} \mathrm{~s}^{-2}$ )

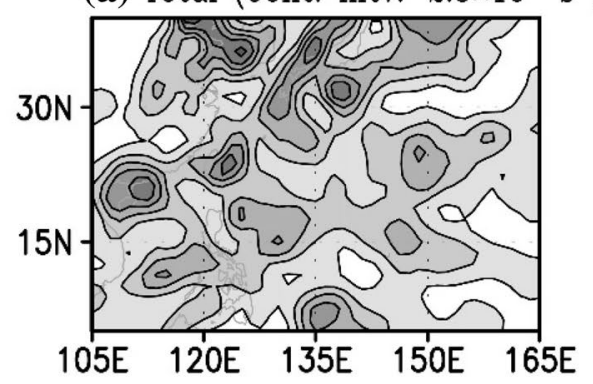

$\zeta_{867}$ variance

(b) noTC (KF, CI $\left.=2.5 \times 10^{-11} \mathrm{~s}^{-2}\right)$

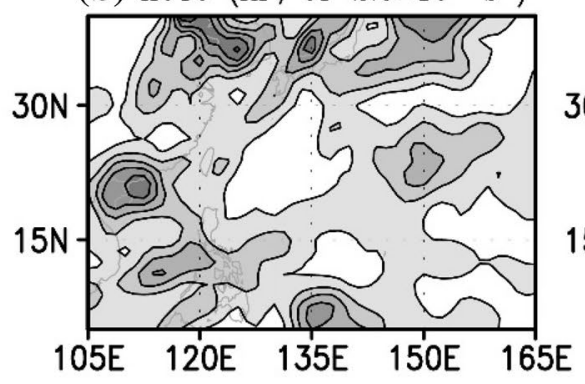

(c) noTC (GDF, CI=2.5 $\times 10^{-11} \mathrm{~s}^{-2}$ )

(d) noTC (PVI, CI=2.5 $\times 10^{-11} \mathrm{~s}^{-2}$ )
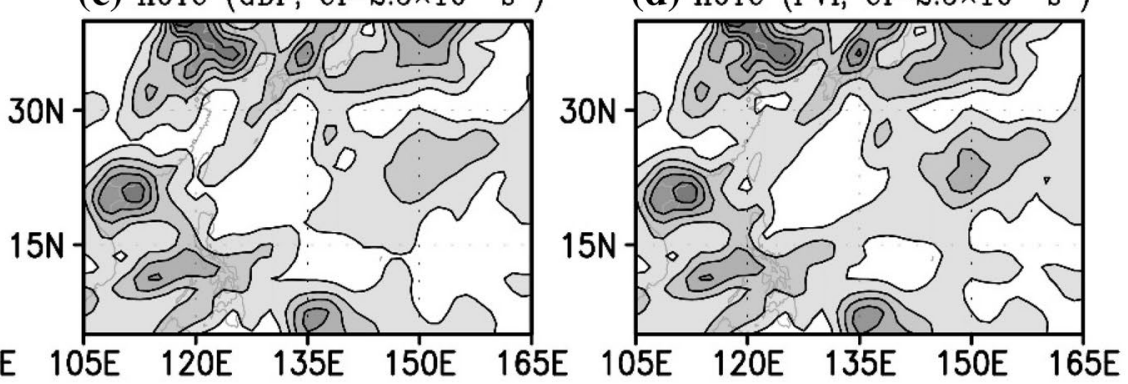

(e) $\mathrm{TC}\left(\mathrm{KF}, \mathrm{CI}=2.5 \times 10^{-11} \mathrm{~s}^{-2}\right)$

(f) $\mathrm{TC}\left(\mathrm{GDF}, \mathrm{CI}=2.5 \times 10^{-11} \mathrm{~s}^{-2}\right)$
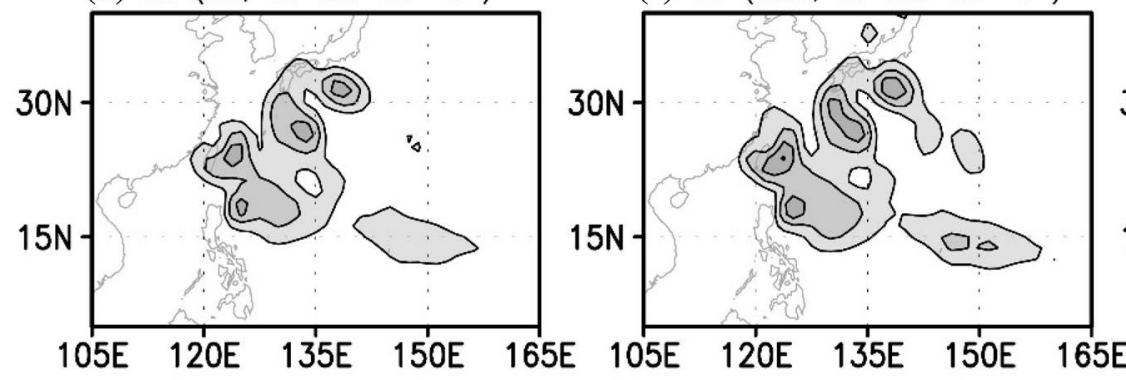

(g) TC (PVI-KF, CI=0.25 $\left.\times 10^{-11} \mathrm{~s}^{-2}\right)$
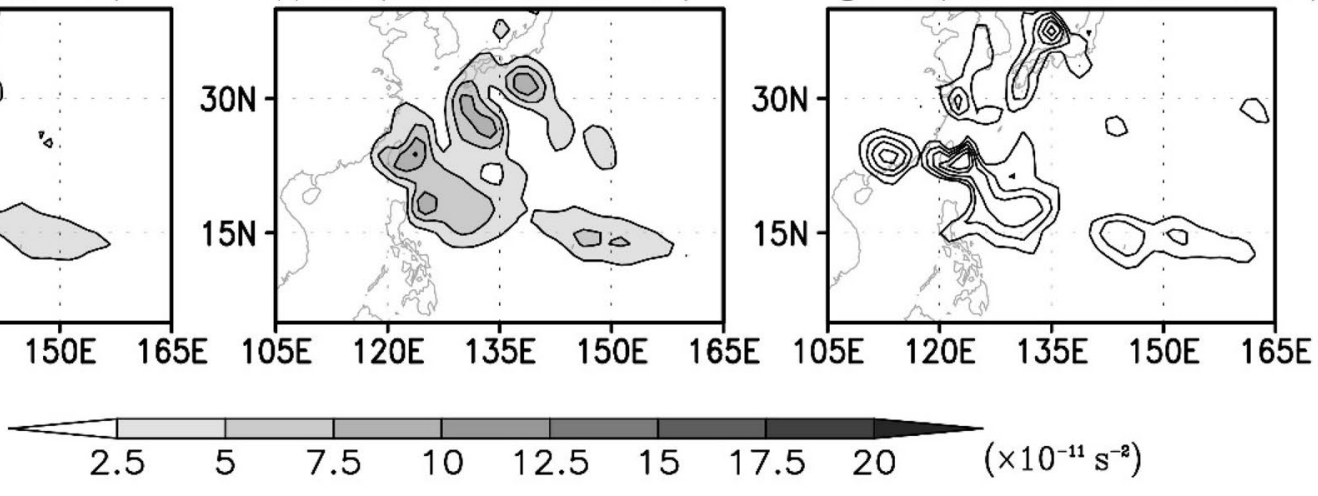

Fig. 14 Intraseasonal (32-76-day) variance of relative vorticity (shade and contour) at $866.9 \mathrm{hPa}$. a JRA-55 reanalysis. b-d TC removed field determined by KF, GDF and PVI, respectively. The difference between $\mathbf{a}$ and $\mathbf{b}, \mathbf{c}$ are shown in $\mathbf{e}, \mathbf{f}$, respectively (referred as

\section{Conclusion and discussion}

By removing TC vortices in the global analysis and by evaluating the resultant differences, one may investigate the effect of TCs on large-scale environmental fields. Although a method to remove TC vortex proposed by Kurihara et al. (1995) is widely used, it is unclear whether the TC-removed field obtained by the existing method is well-balanced. Here a methodology for constructing well-balanced TC-removed fields based on PVI is proposed.

The proposed TC removal technique is unique in several respects. First, owing to the use of PVI, the obtained TC-removed fields at all levels are well balanced in the the TC component of intraseasonal variance). $\mathbf{g}$ The difference of the TC component of intraseasonal variance between PVI and KF. Nonpositive contour is omitted in the bottom panel. Contour interval is $2.5(0.25) \times 10^{-11} \mathrm{~s}^{-2}$ in $\mathbf{a}-\mathbf{f}(\mathbf{g})$

framework of PVI system. Second, although an averaging field is predefined when PVA associated with TC is decomposed from the large-scale field, the effect of the TC-associated PVA is still included in the averaged field if calculated as a simple time mean. The averaged field in the proposed technique is iteratively calculated using the TC-removed fields to reduce the TC residues in the averaged field. Third, the PVA associated with a TC is detected objectively at all layers, and the domain is not necessarily circular. In addition, a vertical vortex axis is defined, which prevents misinterpreting a PVA associated with other synoptic systems as the targeted TC perturbation. Thus, this algorithm does not extract PVAs associated with other synoptic systems that exist in the vicinity of the TC. 


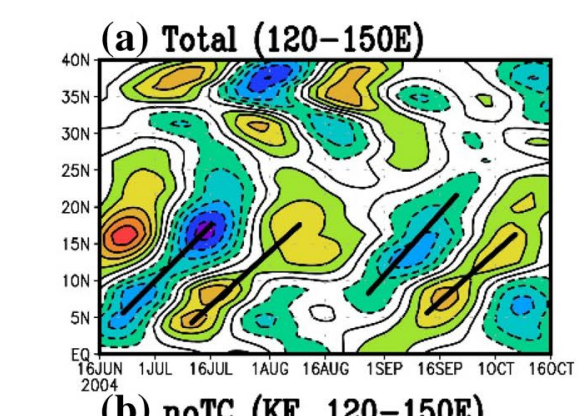

\section{Hovmoller diagram, Averaged $\zeta_{887}$}
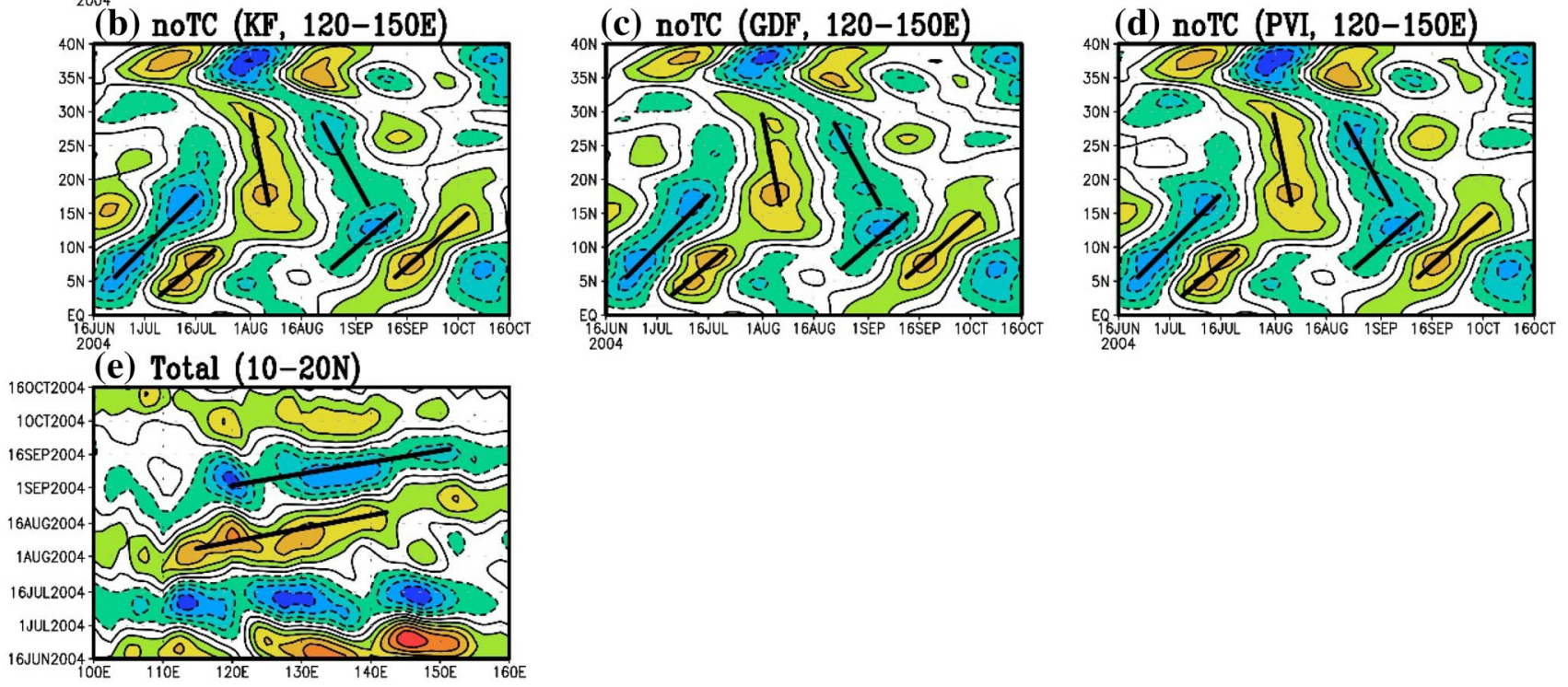

(f) noTC (KF, 10-20N)

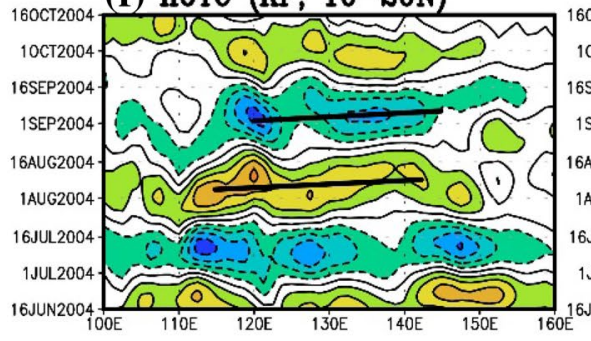

(g) noTC (GDF, 10-20N)

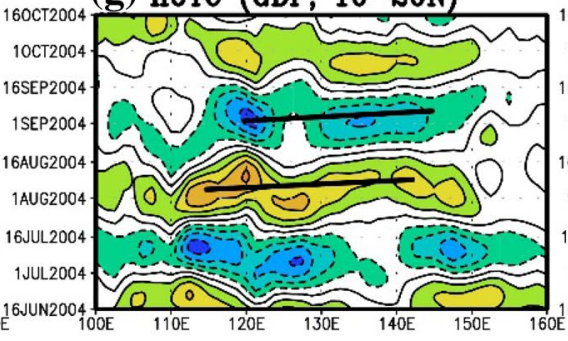

(h) noTC (PVI, 10-20N)

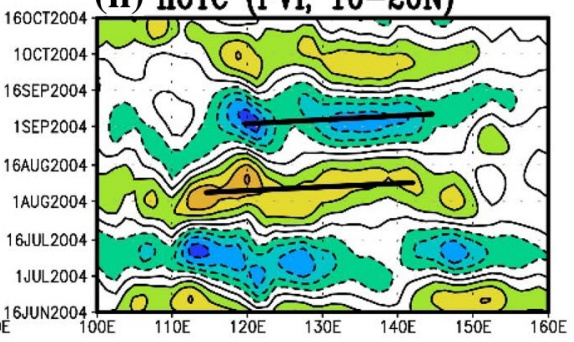

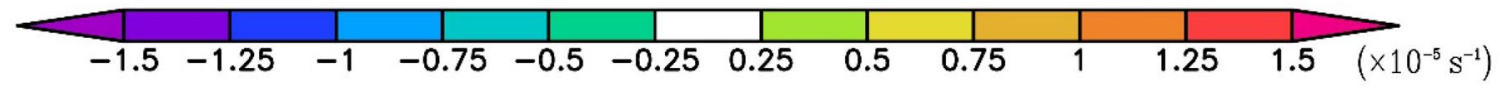

Fig. 15 Hovmöller diagrams of the 32-76-day filtered relative vorticity averaged over $120^{\circ}-150^{\circ} \mathrm{E}$ (color and contour) at $866.9 \mathrm{hPa}$. a JRA-55 reanalysis. b-d TC removed field determined by KF,

The Kurihara method that was popular in TC simulations is compared with our technique. The magnitude of TC vortex calculated by the Kurihara method is smaller. Furthermore, the Kurihara method generates an unrealistic thick negative vorticity band encircling TC center. In the TC-removed field, the Kurihara method tends to retain a weak cyclonic circulation at the location where the removed TC originally existed. Such a weak cyclonic circulation no longer exists in the TC-removed field obtained by the technique proposed here. Moreover, TC vortices
GDF and PVI, respectively. The lower panel $\mathbf{e}-\mathbf{h}$ is the same as the upper panel a-d but averaged over $10^{\circ}-20^{\circ} \mathrm{N}$. Contour interval is $0.25 \times 10^{-5} \mathrm{~s}^{-1}$

obtained by the proposed method have more far-reaching influence than those obtained by the existing method. After the removal of TCs, the environmental field surrounding TCs remains largely unchanged in the existing method, while it changes markedly in the proposed method. In view of the marked change in the environmental field, one might doubt the validity of the proposed TC removal method. In general, TC is embedded in a larger circulation system, for example, the subtropical high and monsoon trough, and is surrounded by non-TC flows. The embedded TC 


\section{PV inversion, 00Z23AUG2004}

\section{(a) Total (Temp., 259.3hPa)}

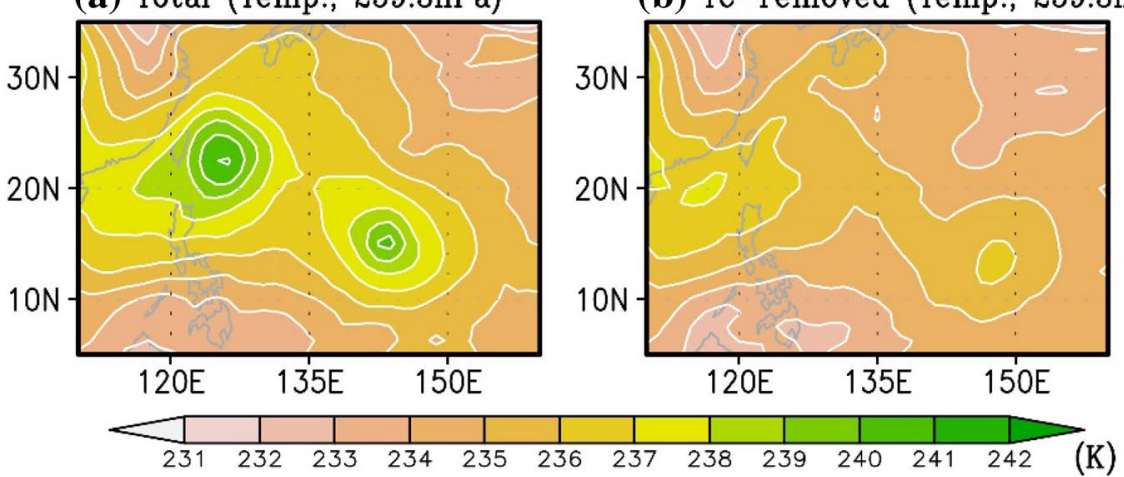

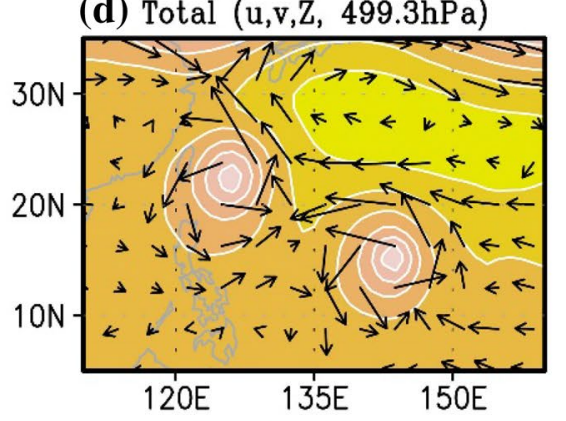

(e) $\mathrm{TC}-$ removed $(\mathrm{u}, \mathrm{v}, \mathrm{Z}, 499.3 \mathrm{hPa})$
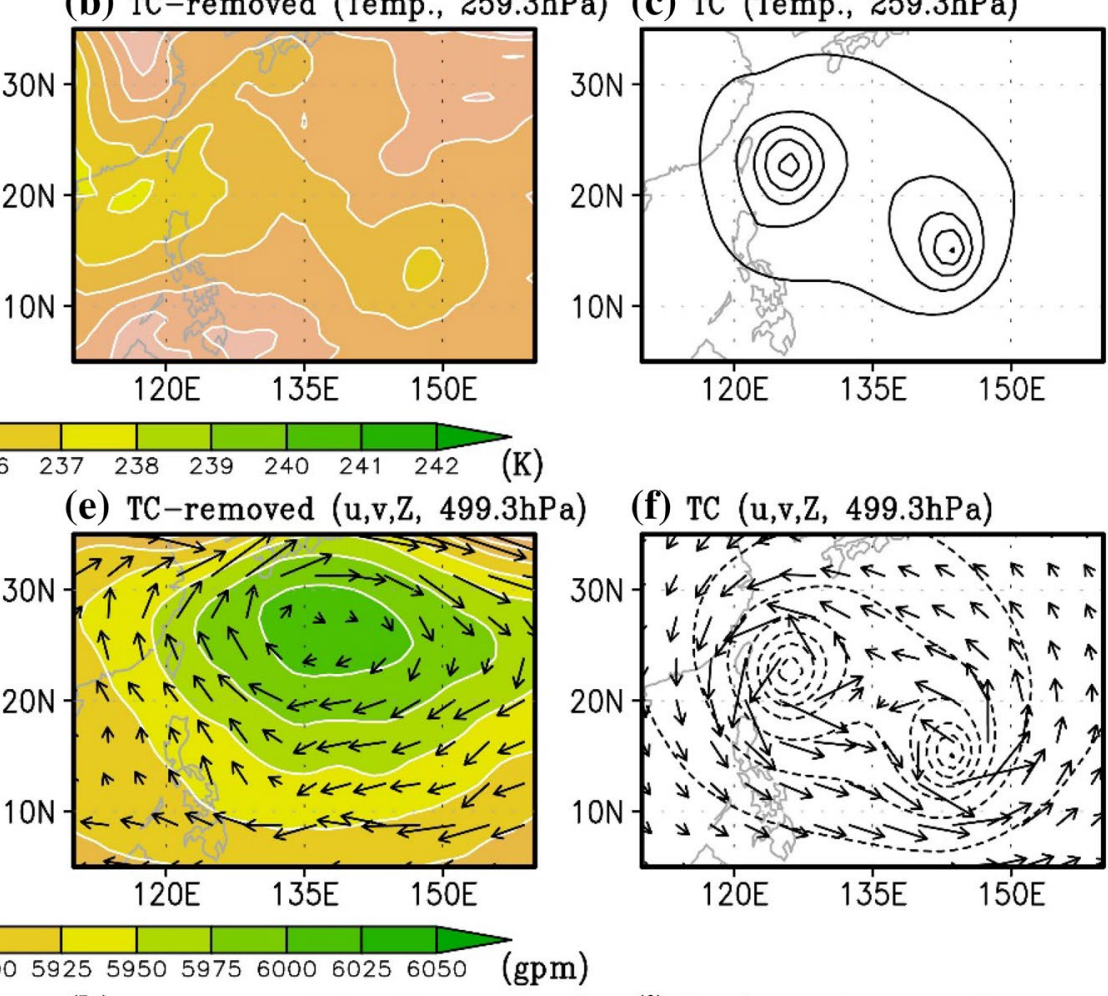

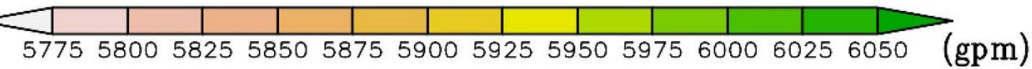

(g) Total $(u, v, Z, 866.9 \mathrm{hPa})$

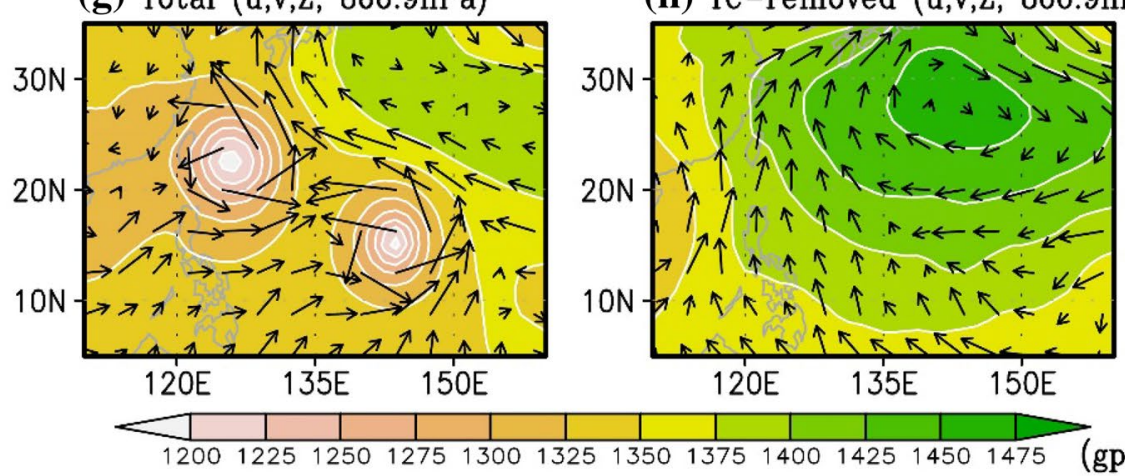

(i) $\mathrm{TC}(\mathrm{u}, \mathrm{v}, \mathrm{Z}, 866.9 \mathrm{hPa})$

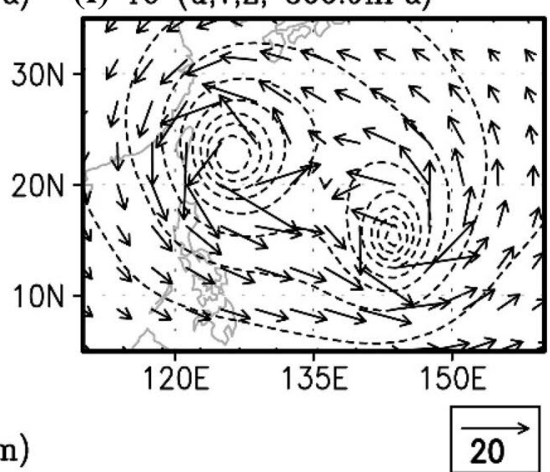

Fig. 16 Same as Fig. 6 but for the results obtained by P-PVI

suppresses these systems because of its strength. In addition, the PV has its dynamic impact on the far field (the so-called electrostatics analogy; Bishop and Thorpe 1994; Thorpe and Bishop 1995). For this reason, it is expected that the derived mass and wind fields change in a region much larger than the TC domain after the removal of PVAs. Therefore, the appearance of marked changes in the environmental fields is not surprising considering the PV dynamics.

A new wind decomposition proposed by Galarneau and Davis (2013) is also compared. The TC contribution to the vorticity fields calculated by GDF is the largest among three methods because GDF extracts not only the TC component of relative vorticity but also background vorticity. Moreover, in comparison with the divergent wind derived by Galarneau and Davis (2013) decomposition, the divergent wind obtained by the balanced omega equation system is smaller (not shown). As reported by Wang and Zhang (2003), this is an inherent problem when the velocity potential and vertical velocity are inverted by the balanced omega equation system. A carefully designed adaptation of Galarneau and Davis (2013) method may help to enhance the underestimated divergent wind in KF and PVI. It would be interesting to construct a new balanced system taking the advantage 


\section{Vertical cross section at $15.00^{\circ} \mathrm{N}, 00 \mathrm{Z} 23 \mathrm{AUG} 2004$}

(a) PN-PVI $\left(q^{\prime}\right)$

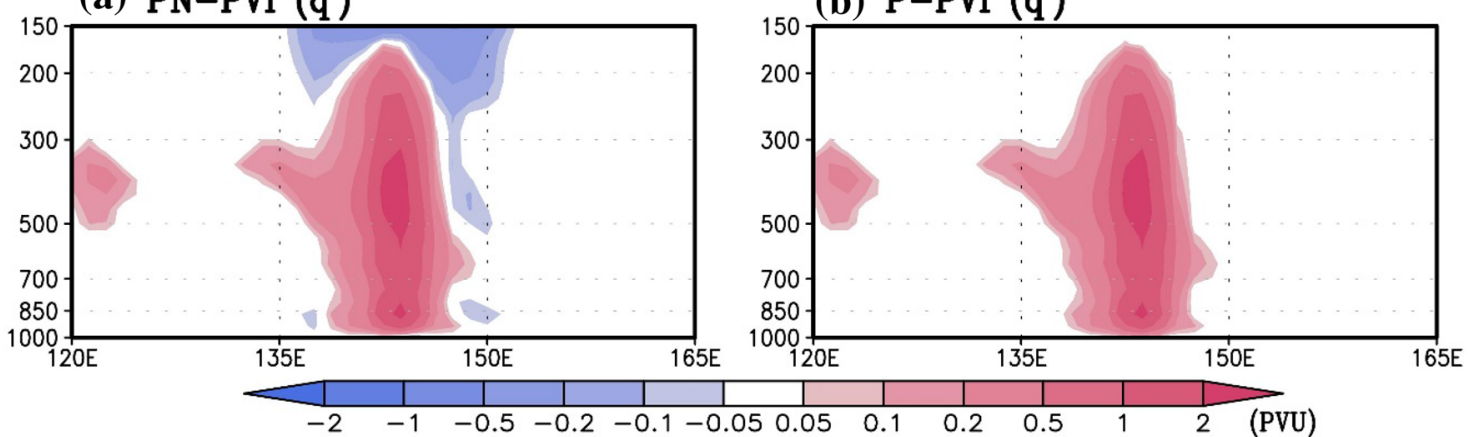

(c) PN-PVI (z')

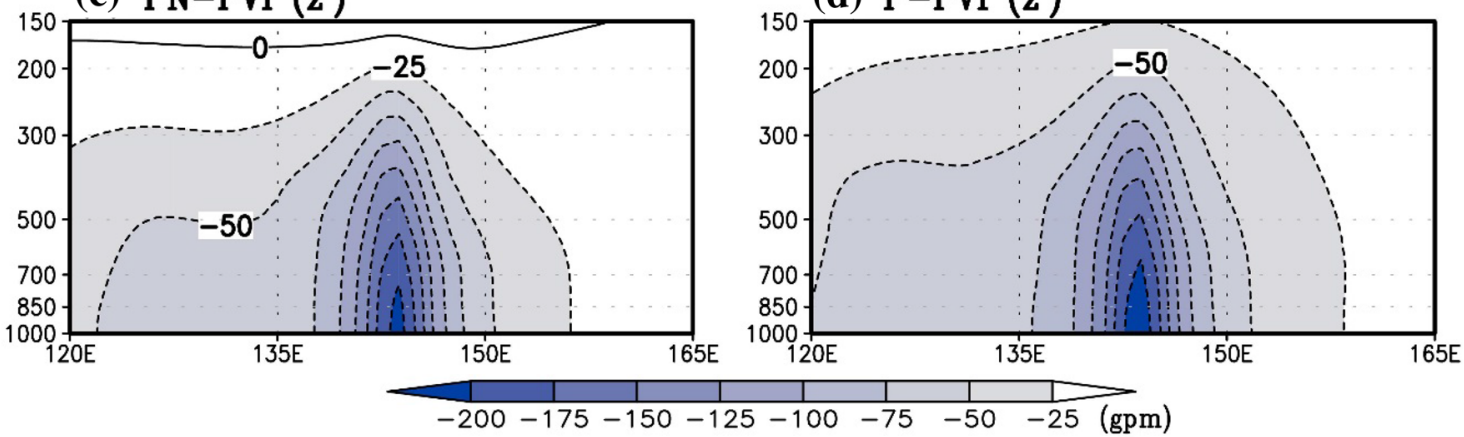

(e) PN-PVI (T")

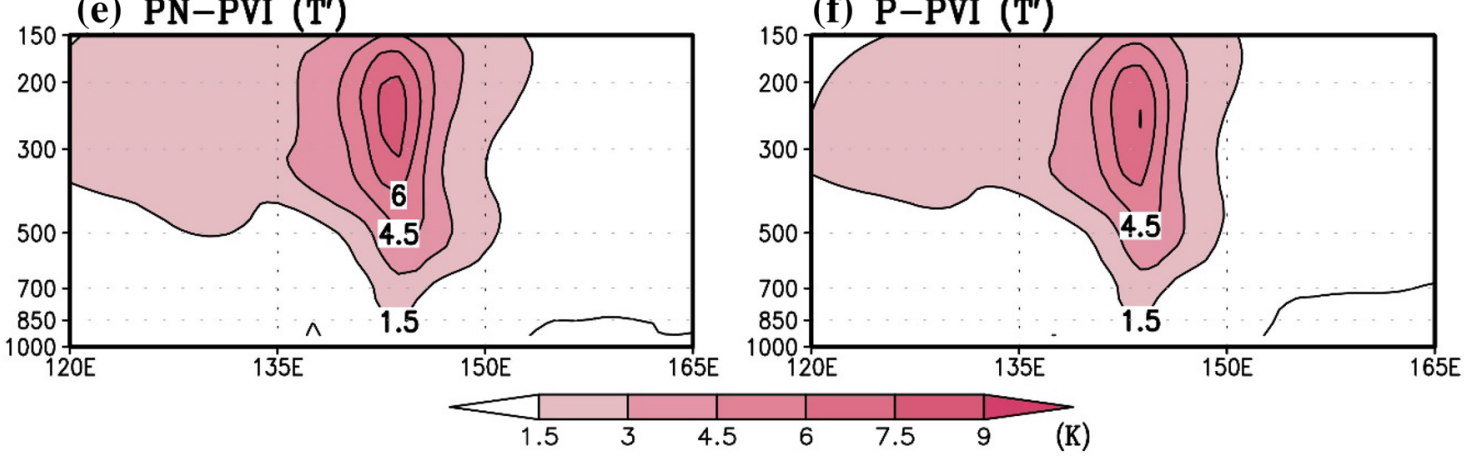

Fig. 17 Vertical cross section at $15^{\circ} \mathrm{N}$ for $0000 \mathrm{UTC}$ on August 23, 2004 of TC-associated (top) PVA, (middle) geopotential height deviation, and (bottom) temperature anomaly. The left-hand (right-hand) column is derived from the result obtained by PN-PVI (P-PVI)

of the method of Galarneau and Davis (2013) to compute more accurate divergent wind and vertical velocity in the TC-removed fields.

$\mathrm{KF}$, GDF and the proposed technique are applied, following Hsu et al. (2008a), to evaluate the TC contribution to the intraseasonal variance and seasonal-mean fields in the tropical WNP during JJASO 2004 and the results are compared. As reported by Hsu et al. (2008a), the summer was characterized by unusually strong TC and intraseasonal activity in the WNP, which reached record high value in 50 years. The results obtained by the proposed method confirm the significant contribution (up to 50\%) of TCs to the intraseasonal variance along the TC tracks revealed by Hsu et al. (2008a). The strong northward-propagating intraseasonal signals were also markedly enhanced by the TCs. The TC contributions calculated by three TC removal schemes are consistent with the results of Hsu et al. (2008a), but the proposed PVI method reveals larger contributions of TCs than KF.

Moreover, although the PV feature associated with TC is characterized by its tropospheric positive anomaly and negative anomaly in the upper troposphere and lower stratosphere, these individual effects are explored. The impact of TC on the large-scale monsoonal flow explored in our study focuses mainly in the lower to middle troposphere where the well-defined positive PV dominates. The "enhanced anticyclonic flow" presented in our study is a direct response to the removal of the positive PV in the lower-middle troposphere. However, the negative PVA has a significant impact in the 
(a) PV anom. (Positive)

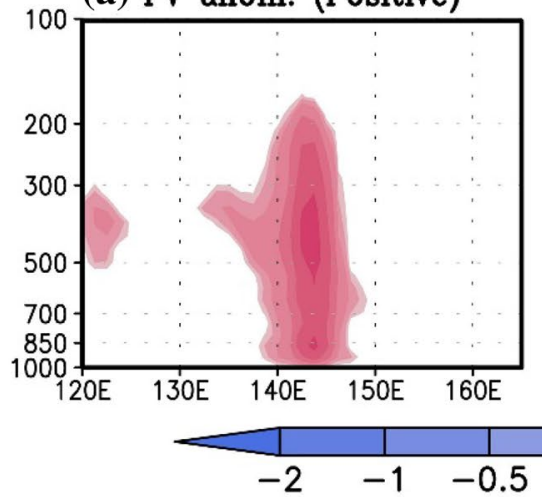

(b) PV anom. (Tropo. Negative)

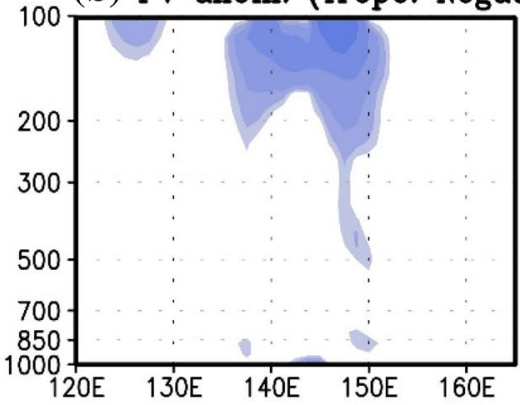

(c) PV anom. (Strat. Negative)

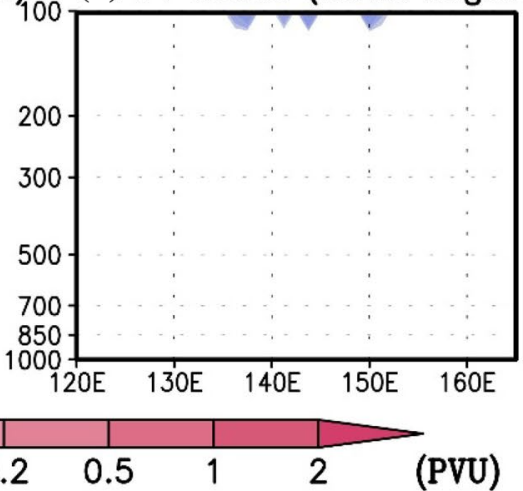

(d) $\mathrm{Z}$ anom.

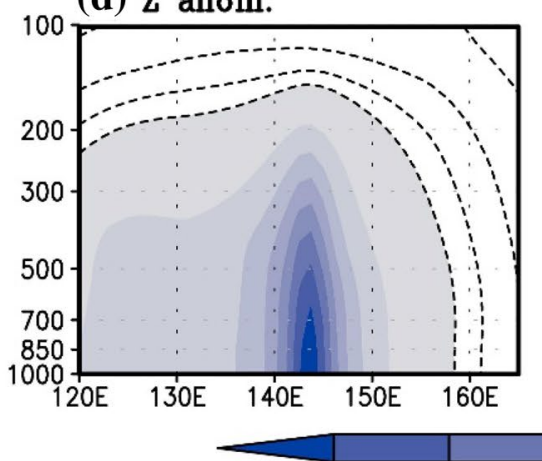

(e) $\mathrm{Z}$ anom.

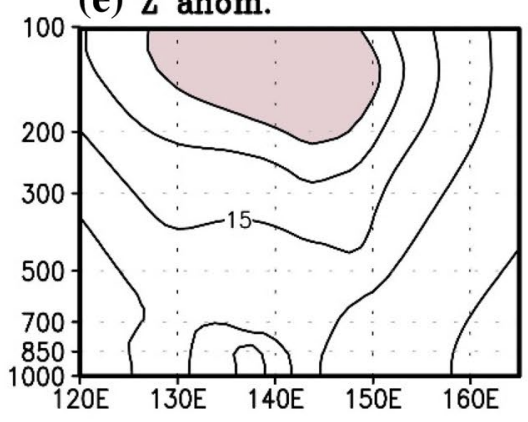

(f) $\mathrm{Z}$ anom.

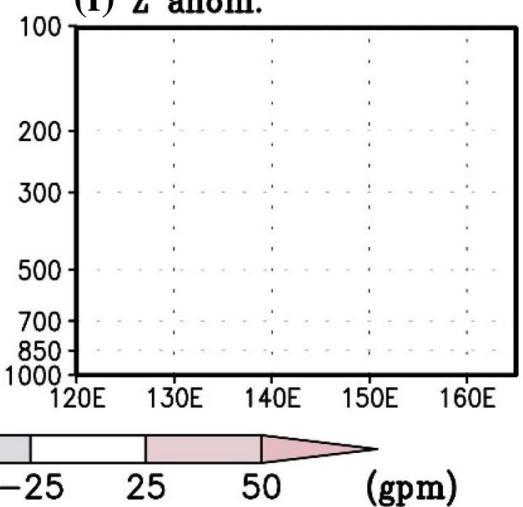

(g) $\mathrm{T}$ anom.

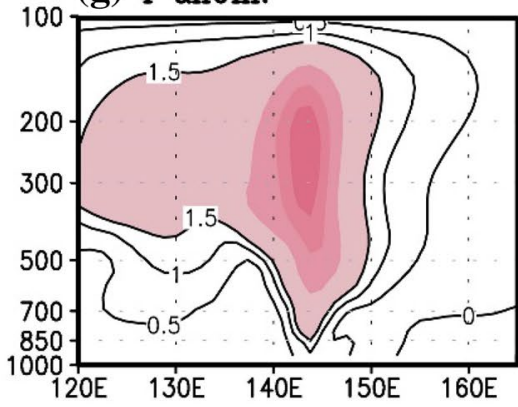

(h) $\mathrm{T}$ anom.

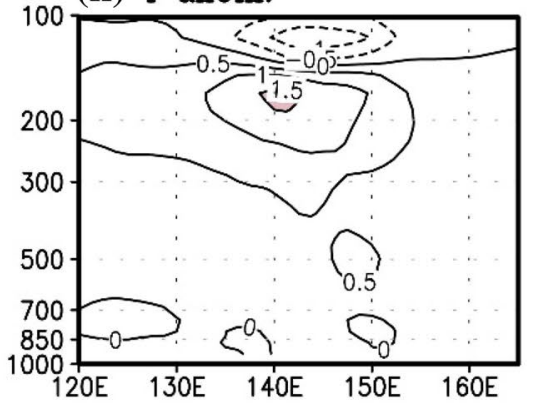

(i) $\mathrm{T}$ anom.

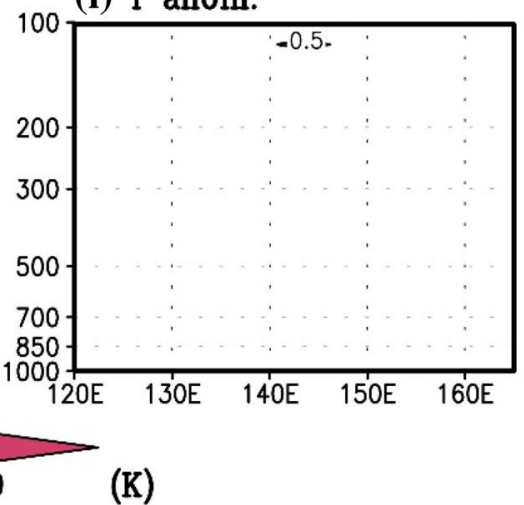

Fig. 18 Vertical cross section at $15^{\circ} \mathrm{N}$ for 0000 UTC on August 23, 2004 of TC-associated a tropospheric positive PVA, d geopotential height deviation (color and contour, contour interval is $5 \mathrm{gpm}$ ranging from -20 to $20 \mathrm{gpm}$ ), and $\mathbf{g}$ temperature anomaly (color and contour, contour interval is $0.5 \mathrm{~K}$ ranging from -1 to $1 \mathrm{~K})$. $\mathbf{b}, \mathbf{e}, \mathbf{g}$ Same as $\mathbf{a}$, $\mathbf{d}, \mathbf{g}$ but for the tropospheric negative PVA. $\mathbf{c}, \mathbf{f}, \mathbf{i}$ Same as $\mathbf{a}, \mathbf{d}, \mathbf{g}$ but for the stratospheric negative PVA upper-level temperature. Thus, we should consider the negative PVA effect when the PVI is applied to TC studies. Due to highly asymmetric distribution of the negative PVA associated with TCs, our definition of the negative PVA associated with a TC in this study is less sophisticated compared with the one of the positive PVA. It would be interesting in the future studies to identify the negative PVA associated with TC with more sophisticated partitioning techniques.
Numerous studies on the multiscale nature of circulation in the tropical WNP have been conducted with respect to energetics in recent years (e.g., Hsu et al. 2011; Ko et al. 2012). Using the data decomposed into different time scales, the aforementioned studies estimated the energy conversion among the fluctuations of different time scales. Despite considering the effect of the presence of TCs on the energetics, the temporal filtering cannot clearly decompose the fields 


\section{3hPa, Temperature}

(a) Total

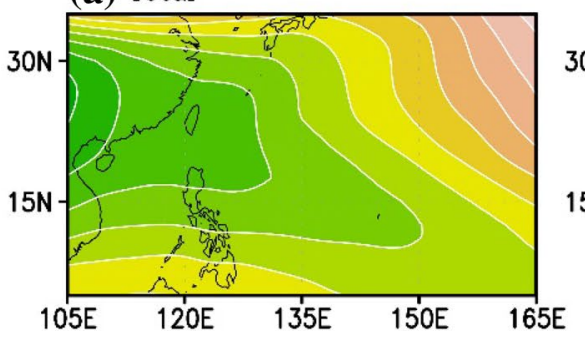

(b) noTC (Positive+Negative)

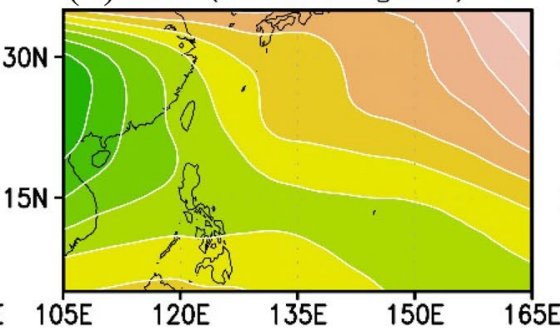

(c) noTC (Positive)

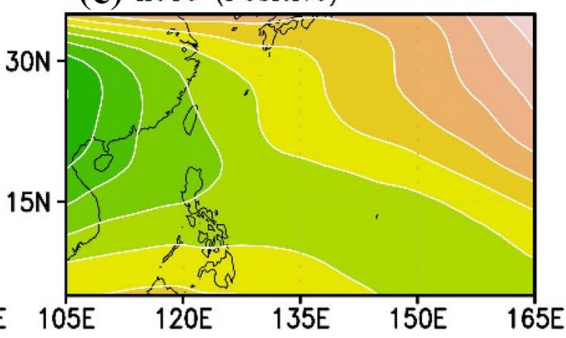

$\begin{array}{lllllllllll}231.5 & 232 & 232.5 & 233 & 233.5 & 234 & 234.5 & 235 & 235.5 & 236 & 236.5\end{array}$

866.9hPa, Geopotential Height

(d) Total

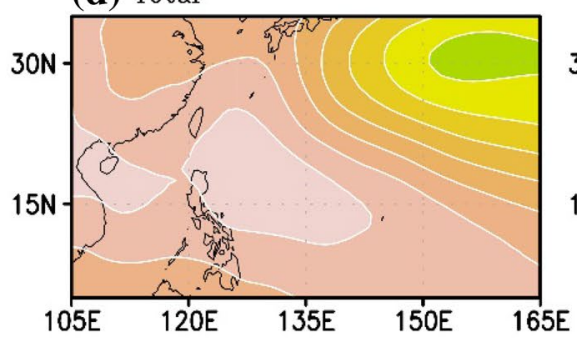

(e) noTC (Positive +Negative)

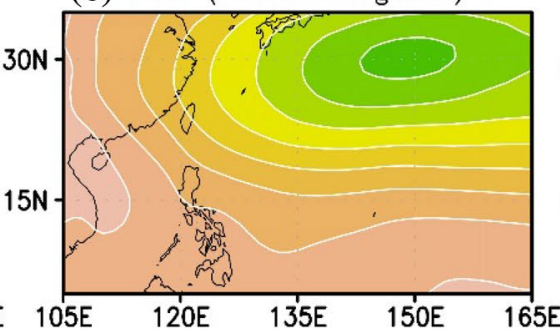

(f) noTC (Positive)

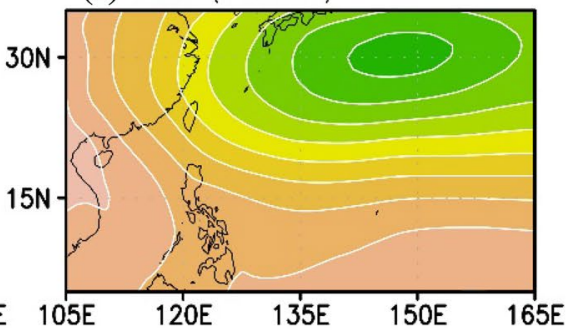

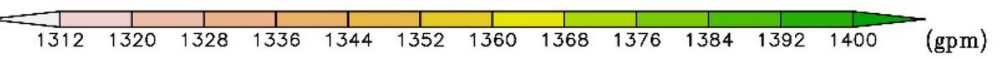

Fig. 19 Temperature (color and contour, in K) at $259.3 \mathrm{hPa}$ for the time mean in JJASO 2004: a JRA-55 reanalysis; TC-removed field obtained by b PN-PVI and $\mathbf{c}$ P-PVI. d-f Same as a-c but for geopotential height at $866.9 \mathrm{hPa}$ (in gpm)

into TC and non-TC components. Clearly, using the TCremoved fields to reveal the TC contribution to the energetics is the preferred way. On the basis of the KF method, Ko et al. (2012) suggested that the presence of TCs enhanced the kinetic energy conversion between submonthly and intraseasonal perturbations by $40-50 \%$. Hsu et al. (2008a) and Ko et al. (2012) analyzed the TC effects at 850-hPa level only because of the limitation of the KF method applied; therefore, they could not reveal the complete characteristics of energy conversion in three dimensions. Furthermore, considering the larger TC amplitude derived from the proposed PVI technique, Ko et al. (2012) might have underestimated the TC contribution. Both TC and TC-removed fields obtained by the proposed removal technique enable the analysis at all layers while retaining dynamic balance. Diagnostics based on such data are expected to obtain a more accurate estimation of energy conversion and dynamical processes involved in the multiscale interaction.

Another challenging task is to evaluate the remote effects of TCs on the large-scale circulation, such as the predecessor rain event (Galarneau et al. 2010; Galarneau 2015) and the downstream effect during the extratropical transition process (Riemer and Jones 2010; Grams and Blumer 2015) through the Rossby wave energy dispersion. This remote effect cannot be realized through the TC removal procedure. Numerical modeling studies with and without TCs are needed for such an impact evaluation.

Acknowledgements The authors acknowledge the reviewer whose comments improved this manuscript. The authors also would like to thank Nagio Hirota of Center for Global Environmental Research, National Institute for Environmental Studies, Japan for his helpful comments on the early version of the manuscript. This study is supported by The Ministry of Science and Technology, Taiwan, under MOST 107-2119-M-001-010 and 108-2119-M-001-014.

Open Access This article is licensed under a Creative Commons Attribution 4.0 International License, which permits use, sharing, adaptation, distribution and reproduction in any medium or format, as long as you give appropriate credit to the original author(s) and the source, provide a link to the Creative Commons licence, and indicate if changes were made. The images or other third party material in this article are included in the article's Creative Commons licence, unless indicated otherwise in a credit line to the material. If material is not included in the article's Creative Commons licence and your intended use is not permitted by statutory regulation or exceeds the permitted use, you will need to obtain permission directly from the copyright holder. To view a copy of this licence, visit http://creativecommons.org/licenses/by/4.0/. 


\section{References}

Bishop CH, Thorpe AJ (1994) Potential vorticity and the electrostatic analogy: quasi-geostrophic theory. Q J R Meteorol Soc 120:713731. https://doi.org/10.1002/qj.49712051710

Cha D-H, Wang Y (2013) A dynamical initialization scheme for realtime forecasts of tropical cyclones using the WRF Model. Mon Weather Rev 141:964-986. https://doi.org/10.1175/MWR-D-1200077.1

Cheung KKW, Chan JCL (1999) Ensemble forecasting of tropical cyclone motion using a barotropic model. Part I: Perturbations of the environment. Mon Weather Rev 127:1229-1243. https://doi. org/10.1175/1520-0493(1999)27\%3c1229:EFOTCM\%3e2.0.CO;2

Davis CA, Emanuel KA (1991) Potential vorticity diagnostics of cyclogenesis. Mon Weather Rev 119:1929-1953. https ://doi.org/10.1175/1520-0493(1991)119\%3c1929:PVDOC \%3e2.0.CO;2

DeMaria M (1996) The effect of vertical shear on tropical cyclone intensity change. J Atmos Sci 53:2076-2088. https ://doi.org/10.1175/1520-0469(1996)053\%3c2076:TEOVS $\mathrm{O} \% 3 \mathrm{e} 2.0 . \mathrm{CO} ; 2$

Franklin JL, Feuer SE, Kaplan J, Aberson SD (1996) Tropical cyclone motion and surrounding flow relationships: searching for beta gyres in omega dropwindsonde datasets. Mon Weather Rev 124:64-84. https://doi.org/10.1175/1520-0493(1996)124\%3c006 4:TCMASF\%3e2.0.CO;2

Galarneau TJ (2015) Influence of a predecessor rain event on the track of Tropical Cyclone Isaac (2012). Mon Weather Rev 143:33543376. https://doi.org/10.1175/MWR-D-15-0053.1

Galarneau TJ, Davis CA (2013) Diagnosing forecast errors in tropical cyclone motion. Mon Weather Rev 141:405-430. https://doi. org/10.1175/MWR-D-12-00071.1

Galarneau TJ, Bosart LF, Schumacher RS (2010) Predecessor rain events ahead of tropical cyclones. Mon Weather Rev 138:32723297. https://doi.org/10.1175/2010MWR3243.1

Grams CM, Blumer SR (2015) European high-impact weather caused by the downstream response to the extratropical transition of North Atlantic Hurricane Katia (2011). Geophys Res Lett 42:8738-8748. https://doi.org/10.1002/2015GL066253

Gray WM (1998) The formation of tropical cyclones. Meteor Atmos Phys 67:37-69. https://doi.org/10.1007/BF01277501

Harada Y et al (2016) The JRA-55 reanalysis: representation of atmospheric circulation and climate variability. J Meteor Soc Japan 94:269-302. https://doi.org/10.2151/jmsj.2016-015

Haynes P, McIntyre M (1987) On the evolution of vorticity and potential vorticity in the presence of diabatic heating and frictional or other forces. J Atmos Sci 44:828-841. https ://doi.org/10.1175/1520-0469(1987)044\%3c0828:OTEOV A\%3e2.0.CO;2

Hendricks EA, Peng MS, Fu B, Li T (2010) Quantifying environmental control on tropical cyclone intensity change. Mon Weather Rev 138:3243-3271. https://doi.org/10.1175/2010MWR3185.1

Hsu H-H, Lo A-K, Hung C-H, Kau W-S, Wu C-C, Chen Y-L (2008a) Coupling of the intraseasonal oscillation with the tropical cyclone in the western North Pacific during the 2004 typhoon season. Recent progress in atmospheric sciences: applications to the AsiaPacific region, Liou KN and Chou M-D Eds., World Scientific, 49-65. https://doi.org/10.1142/9789812818911_0003

Hsu H-H, Hung C-H, Lo A-K, Wu C-C, Hung C-W (2008b) Influence of tropical cyclones on the estimation of climate variability in the tropical western North Pacific. J Clim 21:2960-2975. https://doi. org/10.1175/2007JCLI1847.1

Hsu P-C, Li T, Tsou C-H (2011) Interactions between boreal summer intraseasonal oscillations and synoptic-scale disturbances over the western North Pacific. Part I: Energetics diagnosis. J Clim 24:927-941. https://doi.org/10.1175/2010JCLI3833.1

Ko K-C, Hsu H-H, Chou C (2012) Propagation and maintenance mechanism of the TC/submonthly wave pattern and TC feedback in the western North Pacific. J Clim 25:8591-8610. https://doi. org/10.1175/JCLI-D-11-00643.1

Kobayashi $S$ et al (2015) The JRA-55 reanalysis: general specifications and basic characteristics. J Meteor Soc Japan 93:5-48. https://doi. org/10.2151/jmsj.2015-001

Kurihara Y, Bender MA, Tuleya RE, Ross RJ (1995) Improvements in the GFDL hurricane prediction system. Mon Weather Rev 123:2791-2801. https://doi.org/10.1175/15200493(1995)123\%3c2791:IITGHP\%3e2.0.CO;2

Kwon I-H, Cheong H-B (2010) Tropical cyclone initialization with a spherical high-order filter and an idealized three-dimensional bogus vortex. Mon Weather Rev 138:1344-1367. https://doi. org/10.1175/2009MWR2943.1

Kwon HJ, Won S-H, Ahn M-H, Suh A-S, Chung H-S (2002) GFDLtype typhoon initialization in MM5. Mon Weather Rev 130:29662974. https://doi.org/10.1175/1520-0493(2002)130\%3c296 6:GTTIIM\%3e2.0.CO;2

Murakami T (2014) Tropical cyclones in reanalysis data sets. Geophys Res Lett 41:2133-2141. https://doi.org/10.1002/2014GL059519

Riemer M, Jones SC (2010) The downstream impact of tropical cyclones on a developing baroclinic wave in idealized scenarios of extratropical transition. Q J R Meteorol Soc 136:617-637. https ://doi.org/10.1002/qj.605

Ritchie EA, Frank WM (2007) Interactions between simulated tropical cyclones and an environment with a variable Coriolis parameter. Mon Weather Rev 135:1889-1905. https://doi.org/10.1175/ MWR3359.1

Ritchie EA, Holland GJ (1999) Large-scale patterns associated with tropical cyclogenesis in the western Pacific. Mon Weather Rev 127:2027-2043. https://doi.org/10.1175/15200493(1999)127\%3c2027:LSPAWT\%3e2.0.CO;2

Rogers RF, Chen SS, Tenerelli J, Willoughby HE (2003) A numerical study of the impact of vertical shear on the distribution of rainfall in Hurricane Bonnie (1998). Mon Weather Rev 131:1577-1599. https://doi.org/10.1175//2546.1

Ross RJ, Kurihara Y (1995) A numerical study on influences of Hurricane Gloria (1985) on the environment. Mon Weather Rev 123:332-346. https://doi.org/10.1175/15200493(1995)123\%3c0332:ANSOIO\%3e2.0.CO;2

Shapiro LJ (1996) The motion of Hurricane Gloria: a potential vorticity diagnosis. Mon Weather Rev 124:2497-2508. https ://doi.org/10.1175/1520-0493(1996)124\%3c2497:TMOHG A\%3e2.0.CO;2

Sobel AH, Camargo SJ (2005) Influence of western North Pacific tropical cyclones on their large-scale environment. J Atmos Sci 62:3396-3407. https://doi.org/10.1175/JAS3539.1

Thorpe AJ, Bishop CH (1995) Potential vorticity and the electrostatics analogy: Ertel-Rossby formulation. Q J R Meteorol Soc 121:1477-1495. https://doi.org/10.1002/qj.49712152612

Wang X, Zhang D-L (2003) Potential vorticity diagnosis of a simulated hurricane. Part I: Formulation and quasi-balanced flow. J Atmos Sci 60:1593-1607. https://doi.org/10.1175/2999.1

Wang H, Wang Y, Xu H (2013) Improving simulation of a tropical cyclone using dynamical initialization and large-scale spectral nudging: a case study of Typhoon Megi (2010). Acta Meteor Sinica 27:455-475. https://doi.org/10.1007/s13351-013-0418-y

Wang Y, Rao Y, Tan Z-M, Schnemann D (2015) A statistical analysis of the effects of vertical wind shear on tropical cyclone intensity change over the western North Pacific. Mon Weather Rev 143:3434-3453. https://doi.org/10.1175/MWR-D-15-0049.1

Winterbottom HR, Chassignet EP (2011) A vortex isolation and removal algorithm for numerical weather prediction model 
tropical cyclone applications. J Adv Model Earth Syst 3:8. https ://doi.org/10.1029/2011ms000088

Wu C-C, Emanuel KA (1995) Potential vorticity diagnostics of hurricane movement. Part I: A case study of Hurricane Bob (1991). Mon Weather Rev 123:69-92. https://doi.org/10.1175/15200493(1995)123\%3c0069:PVDOHM\%3e2.0.CO;2

Wu C-C, Yen T-H, Kuo Y-H, Wang W (2002) Rainfall simulation associated with Typhoon Herb (1996) near Taiwan. Part I: the topographic effect. Wea Forecasting 17:1001-1015. https://doi. org/10.1175/1520-0434(2003)017,1001:RSAWTH.2.0.CO;2

Wu C-C, Huang T-S, Huang W-P, Chou K-H (2003) A new look at the binary interaction: potential vorticity diagnosis of the unusual southward movement of Tropical Storm Bopha (2000) and its interaction with Supertyphoon Saomai (2000). Mon Weather Rev 131:1289-1300. https://doi.org/10.1175/15200493(2003)131\%3c1289:ANLATB\%3e2.0.CO;2

Wu C-C, Huang T-S, Chou K-H (2004) Potential vorticity diagnosis of the key factors affecting the motion of Typhoon Sinlaku (2002). Mon Weather Rev 132:2084-2093. https://doi.org/10.1175/15200493(2004)132\%3c2084:PVDOTK\%3e2.0.CO;2

Publisher's Note Springer Nature remains neutral with regard to jurisdictional claims in published maps and institutional affiliations. 